(Aus dem physiol. Institut der Universität Würzburg.)

\title{
Beiträge zur allgemeinen Muskel- und Nervenphysiologie.
}

\author{
II. Mittheilung. \\ Teber die Unentbehrlichkeit von Natrium- (oder Lithium-)lonen \\ für den Contractionsact des Muskels. \\ Von \\ E. Overton.
}

Gegen Ende des Frühlings vom vorigen Jahre (1901) wollte ich hei gewissen osmotischen Untersuchungen mit Muskeln die Lösungen von Elektrolyten völlig vermeiden und dachte statt deren Lösungen von Rohrzucker zu verwenden, die mir sonst (d. h. bei anderen Gewebezellen) in ähnlichen Fällen die besten Dienste geleistet hatten. Es galt natürlich zunächst, einen Vorversuch zu machen, der darin hestand, einen Muskel aus $0,5^{\circ} 0_{0} \mathrm{NaCl}$ in eine ungefär isosmotische Rohrzuckerlösung $\left(6^{0}: 0\right)$ zu übertragen und das Verhalten des Muskels zu beobachten.

Der Muskel gerieth in dieser Zuckerlösung während der ersten Ninuten in rasch auf einander folgende "spontane“ Zuckungen, kam aber bald wienter zu Ruhe, ohne zunächst in seiner Erregbarkeit irgendwie gelitten zu haben. Nach kuzer Zeit indessen wurde der Muskel zu meinem grössten Erstaunen völlig unerregbar, obs.leich er im Wešentlichen ein normales Aussehen behielt und, wie die Wägung in Uebereinstimnıng mit meinen ursprünglichen Erwartungen ergab, keine merklichen Mengen rou Rohrzucker in seine Fasern aufgenommen baben konnte.

Ich war durch dieses Verhalten des Muskels zunächst vollständig verblüft, indem dasselbe mit meinen sonstigen Erfahrungen über die Wirkungen von Rohrzuckerlösungen von gleichen oder selbst höheren Concentrationen auf die verschiedensten Gewebezellen in 
gänzlichem Widerspruch stand. An eine narkotische Wirkung des Rohrzuckers konnte nicht gedacht werden, aber auch eine eigentliche Giftwirkung schien fast ebenso schwer anzunehmen. - Ich begab mich einige Stunden in's Freie, um über das dargebotene Räthsel nachzusinnen, und nach einigen wilden Frklärungsversuchen, die einer ruhigeren Ueberlegung nicht Stand halten konnten, brachte ich eine Arbeitshypothese über die Erscheinung zurück, die, wie gleich vorweg genommen werden mag, zwar nicht die richtige Lösung des Räthsels traf, wohl aber zu Versuchen Veranlassung gab, die den Sachverhalt später aufdeckten.

Der ursprüngliche Gedankengang dieser Hypothese war etwa folgender: Es besteht eine grosse Wabrscheinlichkeit, dass die Muskeln fast ausschliesslich als Elektricitätsleiter zweiter Classe anzusehen sind (bis zu einem gewissen, wenn auch noch so geringem Grade sind vielleicht alle festen und flüssigen Körper Elektricitätsleiter erster Classe, doch ist die Leitung dieser Art bei den nichtmetallischen Körpern mit einzelnen Ausnahmen, wie Kohle, nach den bisherigen Erfahrungen eine so geringe, dass sie für gewöhnlich vernachlässigt werden kann). Nun ist mit der Elektricitätsleitung zweiter Classe stets eine Wanderung der Ionen verbunden. Die lebenden Muskelfasern sind aber wenigstens im Ruhezustand für die meisten Salze undurchlässig, was im besonderen für die Salze des Blutplasmas einerseits und die der Muskelfasern andererseits gilt. Letzteres geht schon daraus hervor, dass die Concentrationen der Salze des Blutplasmas und der Muskelfasern sich während der ganzen Dauer des Lebens nie ausgleichen, die Muskelfasern vielmehr fast frei von Natrium und Chlor bleiben, das Blutplasma andererseits nur Spuren von den in den Muskelfasern so reichlich enthaltenen Kalium und Phosphaten aufweist. Wenn aber die Muskelfasern für diese Salze. undurchlässig sind, so kann auch keine Ionenwanderung durch dieselben stattfinden, und die Elektricitätsleitung (zweiter Classe) müsste auf die Zwischenflüssigkeit beschränkt bleiben. Beim Ersatz des' natürlichen Zwischenflüssigkeit durch die Lösung eines Nichtleiters schien es also verständlich, dass eine elektrische Reizung des Muskels ohne Erfolg blieb, da durch diesen Ersatz der elektrische Widerstand der Muskeln ausserordentlich stark zunehmen und die Stromintensität im gleichen Grade abnehmen müsste.

Ein Einwand gegen diese Auffassung, auf den ich später zurückkommen werde, war mir allerdings gleich bei ihrer Entwicklung 
nicht gänzlich entgangen, doch wurde derselbe zunächst durch eine scheinbare Bestätigung der Hypothese zurückgedrängt.

Den Anstoss erregenden Muskel hatte ich zum Glück nicht weggeworfen, sondern in der Zuckerlösung liegen gelassen. Bei meiner Zurückkehr in's Laboratorium setzte ich der Zuckerlösung, nachdem ich mich noch einmal von der völligen Unerregbarkeit des Muskels überzeugt hatte, eine geringe Menge einer stärkeren $(2,40 \%$ igen $)$ Kochsalzlösung hinzu, so dass einerseits eine grössere Concentrationsänderung der Zuckerlösung, andrerseits eine zu starke wasserentziehende Wirkung der Lösung vermieden wurde. Zu meiner grossen Genugthuung kehrte in dieser etwas modificirten Lösung die Erregbarkeit des Muskels bei der allmählichen Diffusion des Natriumchlorids in die Zwischenflussigkeit des Muskels in der That zurück, und nach einiger Zeit war die Contraction fast so stark und bei einem nur wenig geringeren Rollenabstand auszulösen als bei einem normalen Muskel.

Schon bei der ersten Conception der Hypothese wurde die Consequenz gezogen, dass, wenn sie richtig sei, ein Nervmuskelpräparat, das vollständig in eine Zuckerlösung untergetaucht wird, nach einiger Zeit sowohl direct als auch vom Nerven aus elektrisch unreizbar werden mïsse, dass aber durch nachträgliches Eintauchen eines Nervenabschnittes dieses Präparates in eine Kochsalzlösung von geeigneter Concentration die Auslösung einer Muskelcontraction durch elektrische Reizung des Nerven an dieser Stelle (nicht aber an den übrigen Theilen des Nerven) möglich sein sollte. Die elektrische Reizung des Nerven würde nämlich nur eine elelitrische Leitfähigkcit der Lösung zwischen den Nervenfaseru an der Applicationsstelle der Filektroden, d. h. in den Nervenabschnitt zwischen den Elektrodeu, fordern, denn die wejtere Fortpflanzung der Erregung in den Axencylindern bis $\mathrm{zu}$ den motorischen Endplatten und von hier durch die Muskelfasern ist, wenigstens nach den bisherigen Arssehauungen, von der Beschaffenheit der Lösung zwischen den Nerven- und Muskelfasern völlig unabhängig, sofern diese Lösung keine direct schädigende Wirkung auf die Fasern ausübt.

Nach dem Erfolge des Kochsalzzusatzes beim einfachen Muskelpräparat hegte ich die bestimmte Erwartung, dass dies alles sich genau, wie ausgedacht, ereignen würde, und war so unvorsichtig, ohne zuuächst einen Vorversuch gemacht zu haben, meinen Chef, Prof. v. Frey, zu bitten, dem Versuche beizuwohnen, der in seinem 
zweiten Theile gewissermaassen die Unkehrung der Erscheinungen bei der Curarevergiftung zeigen sollte. Der erste Theil des Versuchs ging ganz nach Programm; das seit längerer Zeit in einer $6^{0}$ oigen Rohrzuckerlösung verweilende Nervmuskelpräparat war direct, wie vom Nerven aus, völlig unerregbar. Es wurde nun das proximale (centrale) Ende des Nerven in eine 0,6\% ige Kochsalzlösung gesetzt, während der Muskel selber und die distale (periphere) Hälfte des Nerven ausserhalb der Lösung in einem mit Wasserdampfe gesättigen Raume verweilten. Um der Kochsalzlösung reichlich Zeit zu lassen, in die Flüssigkeit zwischen den Nervenfasern an der proximalen Hälfte des Nerven zu diffundiren, wurde ein weiterer Reizversuch erst nach einer Stunde vorgenommen. Trotzdem konnte nach Verlauf dieser Zeit eine Muskelcontraction ebensowenig bei der Reizung der proximalen wie der distalen Hälfte des Nerven ausgelöst werden. Die proximale Nervenhälfte wurde noch weitere drei Stunden in der Kochsalzlösung gelassen, aber auch nach deren $\mathrm{Ab}$ lauf blieb eine Nervenreizung erfolglos. - Es war schon ziemlich sicher, dass die zunächst gegebene Erklärung der Unerregbarkeit von Muskeln in reinen Zuckerlösungen nicht die richtige war, oder rlass sie wenigstens einer Modification bedarf.

Das Nervmuskelpräparat wurde nunmehr in eine Lösung von $5 \%$. Rohrzucker $+0,2 \%$ Natriumchlorid übertragen, und zwar so, dass das ganze Präparat in die Lösung untertauchte. Nach zwei Stunden contrahirte sich der Muskel ganz normal, sowohl bei directer Reizung als auch bei Reizung von einer beliebigen Nervenstelle ans. Damit war der Nachweis erbracht, dass das Präparat nicht etwa durch die verschiedenen Manipulationen beschädigt worden war.

Von einem zweiten ganz ähnlichen Versuche gebe ich das wörtliche Protokoll: Um 225 p. m. des 19. Juni 1901 Gastrocnemius und Nerv einer jungen Rana esculenta, die zusammen nach 95 Vinuten Verweilen in $0.6 \% \mathrm{NaCl} 89 \mathrm{cg}$ wogen (Gewichtsconstanz noch nicht erreicht, Gewicht in Zunahme begriffen), in $100 \mathrm{ccm} 6 \%$ iger Rohrzuckerlösung übergefübrt. In Anfange des Versnchs von allen Punkten des Nerven aus gut reizbar. Der vorher in völliger Puhe befindliche Muskel gerieth in der Zuckerlösung in beständige Zuckungen, die indessen, nachdem sie allmählich seltener geworden waren, nach ca. 10-12 Minaten gänzlich aufhörten. - Um $3.22 \mathrm{p} . \mathrm{m}$. Muskel vom Nerven aus bei $10 \mathrm{~cm}$ (statt $22-25 \mathrm{~cm}$ ) Rollenabstand reizbar, directe Reizbarkeit des Muskels nur noch bei $3 \mathrm{~cm}$ (statt 12 
bis $14 \mathrm{~cm}$ ) und Contraction recht schwach. - Um 4.10 p. in. vom Nerven aus bei 9 , direct erst bei $2 \mathrm{~cm}$ Rollenabstand reizbar und Contraction äusserst schwach. - Um 5.45 p. m. weder vom Nerven aus, noch direct erregbar. - Um 6.00 p. m. eine längere Strecke des Nerven über den Rand des Gefässes gebogen und in eine $0,6 \%$ ige Kochsalzlösung untergetaucht, die sich in einer dicht neben dem Gefäss gestellten Röhre befand, während der Muskel selber und die distale (periphere) Hälfte des Nerven in der Robrzuckerlösung gelassen wurden. Gefäss und Röbre unter eine kleine, feuchte Kammer gestellt. - Um 7.00 p. m. von den versehiedensten Stellen des Nerven aus, wie direct, völlig unreizbar. - Um 9.15 p. m. ebenso unerregbar, 97 cg. - Um 9.17 p. in. das ganze Präparat in $5 \%$ Rohrzucker $+0,2 \% \mathrm{NaCl}$ übertragen. - Um 12.30 a. m. des 20. Juni (also nach $3^{1 / 4}$ Stunden, in der Zwischenzeit nicht untersucht) 921/2 cg, wieder ausgezeichnet erregbar und zwar von allen Punkten des Nerven aus bei $18-22 \mathrm{~cm}$ Rollenabstand, Muskel direct bei $11 \mathrm{~cm}$; Contraction sowohl vom Nerven aus, wie direct, recht stark und lebbabt. - Um 8.05 a. m. des 20 . Juni vom Nerven aus bei ca. 15-18, direct bei 9-10 cm reizbar. - Um 10.00 a. m. des 21 . Juni $86 \mathrm{cg}$, nur spurweise erregbar. Versuch abgebrochen.

Um nun zu exfahren, ob bei Nervmuskelpräparaten, die in kochsalzfreien Rohrzuckerlösungen verweilt haben, bloss die Functionen der Muskeln geändert, oder ob zugleich die Nervenstämme beeinflusst werden, wurde ein weiteres Nervmuskelpräparat (Gastrocnemius + Ischiadicus) so aufgehängt, dass nur der Nervenstamm (dieser aber bis zu seiner Eintrittsstelle in den Musikel) in eine $6 \%$ ige Rohrzuckerlösung untertauchte, während der Muskel selber im dampfgesăttigten Raum oberhalb der Lösung blieb. Besondere Acht wurde darauf gegeben, dass der Nervenstamm wirklich in der Lösung untergetaucht blieb, nicht etwa bloss an der Oberfläche der Lösung fluthete. Der Versuch ergab, dass selbst nach 12 Stunden der Muskel, gerade wie unter normalen Umständen, schon bei einem viel grösseren Rollenabstende vom Xerven aus, und zwar vom proximalsten (centralsten) Theil desselben in Erregung zu rersetzen war als bei der directen Muskelreizung. Die Zuckerlösung schien also gänzlich ohne Einfluss auf den Nervenstamm zu sein. Eine gıössere Anzahl weiterer Versuche in dieser Richtung zeigte, dass die Nerven in $6-7 \%$ igen Rohrzuckerlösungen ebenso lange und ebenso leicht reizhar bleiben wie in $0,6-0,7 \%$ igen Kochsalzlösungen. 
Aus diesen Versuchen war zu entnehmen, dass die Erklärung für den eigenthümlichen unerregbaren Zustand der Muskeln in salzfreien Rohrzuckerlösungen keine ganz einfache ist, und dass sie nur von einer systematischen experimentellen Untersuchung zu erhoffen sein würde. Mehrere Versuchsreihen, die das Problem von verschiedenen Seiten aus angriffen, wurden daher gleichzeitig in Gang gesetzt; doch dürfte es zweckmässiger sein, hier die zeitliche Folge der einzelnen Versuche zu unterbrechen und bei der Darstellung der Versuchsergebnisse so vorzugehen, dass die Hauptfragen, die sich an die Erscheinung knüpfen, soweit dies bisher möglich ist, eine nach der anderen ihre endgültige Entscheidung finden.

Zunächst sind indessen einige allgemeine Bemerkungen über das Versuchsmaterial und die Versuchsanordnung vorauszuschicken.

Schon sehr frühzeitig wurde festgestellt, dass alle quergestreiften Froschmuskeln (incl. der Herzmuskulatur) sich in Zuckerlösungen im Wesentlichen gleich verhalten und nur selbstverständliche Unterschiede bezüglich der Zeitdauer, die zum Eintreten des unerregbaren Zustandes erforderlich ist, aufweisen. In der Folge wurden danu vorwiegend der Gastrocnemius meist in Verbindung mit den möglichst schonend präparirten Ischiadicus aber ohne Knochenansatz, der Sartorius, der M. cutaneus pectoris und die Hinterfussmuskeln, letztere in situ, zu den Versuchen verwendet. Die Versuche sind nunmehr zu allen Jahreszeiten ausgeführt worden und geben stets dieselben Resultate, nur dass namentlich der Sartorius die complicirteren Versuche viel besser im Herbst als im Frühling und in Sommer aushält.

Bezüglich der Wägungsversuche, die hier nur zur Controle dienten, ist zu bemerken, dass sie nicht mit derselben Ganauigkeit ausgeführt wurden, wie bei den Versuchen in der ersten Mittheilung. Namentlich verweilten die Muskeln vor Anfang der eigentlichen Versuche meist zu kurze Zeit in den Kochsalzlösungen, um (praktiseh) vollständige Gewichtsconstanz erreicht zu haben. Ich liess die Muskeh gewöhnlich auch nur abtropfen vor der Wägung, statt sie an Fliesspapier abzutrocknen, was, wie ich später fand, die Genauigkeit der Wägungsversuche etwas beeinträchtigt. Deraus erklärt sich z. B., dass die Muskeln bei der Ueberführung aus $0,6 \% \mathrm{NaCl}$ in $6 \%$ Rohrzucker häufig kaum eine Gewichtsabnahme zeigten, und dass die relativen Gewichtsänderungen nicht immer genau dieselben sind. Es schien mir eben wünschenswerth, die Muskeln möglichst bald nach ihrer Präparation den eigentlichen Versuchen zu unterwerfen. 
Dass die Muskeln zunächst meist in eine $0,5^{0} ; 0$ ige $\mathrm{NaCl}$ statt in eine 0,65 oder $0,7^{\circ}$;o ige NaCl-Lösung gebracht wurden, gesehah in der Absicht, dass hei den weiteren Manipulationen mit den Muskeln die Wasserbewegung stets in demselben Sinne erfolgte, und dass die Wasserentziehung niemals einen schädlichen Grad erreichen komnte.

\section{Ist die Unerregbarkeit der Mnskehn nach längerem Ver-} weilen in einer Rohrzuckerlösung dnrch eine specifisch schädliche Wirkung des Rohrzuckers oder durch den Mangel an Natriumchlorid in der Zwischenflüssigkeit bedingt?

Schon die Vorversuche deuteten darauf hill, dass die Unerregbarkeit der Muskeln nicht durch eine besondere Giftwirkung des Rohrzuckers bewirkt merden konnte, indem die Zuckerconcentration nur wenig erniedrigt w'urde durch den nachträglichen Zusatz einer Kochsalzlösung. Inmerhin werden wir in der dritten Mittheilung Fälle kennen lernen, wo eine Verbindung in einer Concentration $c$ unschädlich ist, ja günstị wirkt, in einer wenig höheren Concentration $e^{\prime}$ dagegen völlig lähmend. Um nun diese Frage bezüglich des Rohrzuckers zu entscheiden, wurden zwei Wege eingeschlagen. Einmal wurden Muskeln in 4-, 5- und 6\% ige Lösungen von Rohrzucker ohne $\mathrm{Zusatz}$ von $\mathrm{NaCl}$ und andere Muskeln in gleich concentrirte Rohrzuckerlösungen mit einer Zugabe von $0,1-0,15 \% \mathrm{NaCl}$ gesetzt, einmal wurde der Rohrzucker durch isosmotische Lösungen von anderen Non-Elektrolyten ersetzt.

In der ersten Reihe von Versuchen kamen die Muskeln bisweilen von vorn herein in Lösungen, die neben $4,5,6 \%$ Rohrzucker noch $0,1-0,15 \% \mathrm{NaCl}$ enthielten, bisweilen kamen sie zuerst in salzfreie Rohrzuckerlösungen, die erst nachträglich einen Zusatz von festem $\mathrm{NaCl}$ erfuhren.

Diese Versuche ergaben, dass alle Froschmuskeln in den 4, 5und $6 \%$ igen Rohrzuckerlösungen, die von vorn herein 0,1 oder besser $0,15 \% \mathrm{NaCl}$ enthielten, gut erregbar blieben und ebenso lange lebten wie in $0,6 \%$ igen Kochsalzlösungen, dass dagegen Muskeln ebenso unerregbar werden in reinen $4 \%$ igen wie in reinen $6^{\circ}: 0$ igen Rohrzuckerlösungen, wobei aber ein nachträglicher Zusatz von etwas festem Kochsalz zu diesen Lösungen (so dass die partielle Concentration des $\mathrm{NaCl} 0,1-0,15 \%$ hetrug) die Contractionsfähigkeit der Muskeln wieder restaurirte. Speciell bei den Hinterfüssen (am ge- 
eignetsten sind zur Beobachtung die kurzen Zehenmuskeln, wo der unerregbare Zustand in wenigen Minuten eintritt, und wo nach Zusatz von $\mathrm{NaCl}$ die Contractionsfähigkeit ebenso schnell wiederkehrt) können die Muskeln über $2+$ Stunden in dem unerregbaren Zustand verharren, um auf Zusatz des Natriumchlorids ihr Contractionsvermögen wieder zu erlangen.

Von den zahlreichen Versuchen über diesen Gegenstand gehe ich nur einige wenige in stark ahgekürzter Form.

\section{Fersuch 1.}

Um 6.45 p. m. des 23 . Juni 1901 wurde ein Sartorius $(20 \mathrm{cg})$ nach etwas langerem Liegen in $0,6 \% \mathrm{NaCl}$ in $6 \%$ Rohrzucker $+0,1 \% \mathrm{NaCl}$ übertragen. Um $7.58 \mathrm{p}$. m. bei $11 \mathrm{~cm}$ Rollenabstand gut reizbar, $1812 \mathrm{cg}$ - Um $9.22 \mathrm{p.m}$. bei $10 \mathrm{~cm}$ Rollenabstand Contraction kräftig, 181 ia $\mathrm{cg}$. - Un 7.30 a. m. des 24. Juni $18^{1} \cdot 2 \mathrm{cg}$, bei $9-10 \mathrm{~cm}$ Contraction ziemlich stark. - Um $6.00 \mathrm{p} . \mathrm{m}$. des 24. Juni noch bei $9 \mathrm{~cm}$ reizhar, Contraction etwas schwach. - Um 11.00 p. m. unerregbar, aber noch nicht todesstarr.

\section{Versucli 2.}

Um 6.50 p. m. des 23. Juni ein Gastrocnemius + Nerv, die nach längerem Liegen in $6^{0} \cdot 0 \mathrm{NaCl}$ (Gewicht noch in Zunahme begriffen) $107 \mathrm{cg}$ wogen, in $6^{0}: 0$ Robrzucter $+0,1 \% \mathrm{NaCl}$ ubertragen. - Um 9.55 p. m. $102 \mathrm{cg}$; vom Nerven aus bei $18 \mathrm{~cm}$, direct bei $9 \mathrm{~cm}$ Rollenabstand contrahirbar. - Um 7.33 a. m. des 24. Juni $103 \mathrm{cg}$, vom Nerven aus bei $13-15$, direct bei $8-9 \mathrm{~cm}$ Rollenabstaud reizbar. - Um 5.20 p. m. des 24 . Juni $103 \%$.2 cg; direct bei $8 \mathrm{~cm}$, rom Nerven aus nicht mehr reizbar.

\section{Versuch 9.}

Um 6.40 p. m. des 25. Juni wurde ein Sartorius, der nach längerem Liegen in $0.6^{\circ} \% \mathrm{NaCl} 22 \mathrm{cg}$ wog in $6^{\circ} \circ \mathrm{Rohrzucker}$ übergeführt. - Um $7.25 \mathrm{p} . \mathrm{m}$. völlig unerregbar. - Um 9.85 p. m. des 25 . Juni $22 \mathrm{cg}$, keine Spur ron Erregbarkeit. - $\operatorname{Cm} 8.08$ a. m. des 26 . Juni $22^{1 / 2} \mathrm{cg}$. - Um 8.32 a. m. in 6\% Rohrzucker + 0.1 ${ }^{\circ} \mathrm{NaCl}$ übertragen. - Um 2.05 p. m. des 25 . Juni $20 \% \mathrm{cg}$, bei 2-3 cm Rollenabstand etwas reizbar, aber Contraction schwach.

\section{Versuch 1.}

Um 7.50 p. m. des 4. Januar 1902 wurde ein Hinterfuss von Rana esculenta, deren kurze Zehenmuskeln bei $8-10 \mathrm{~cm}$ Rollenabstand gut contrahirbar waren, in $6^{\circ}$ :o Rohrzucker übergeführt. - Um $8.01 \mathrm{p}$. m. kurze Nuskeln der mittleren Zehe noch bei $5 \mathrm{~cm}$ Rollenabstand reizbar, aber Contraction recht träge, die kurzen Muskeln der übrigen Zeben schon völlig unerregbar selbst bei völlig genäherten Rollen. - Um 8.07 p. m. des 4. Januar auch die kurzen Muskeln der mittleren Zehe unerregbar. - Um 5.42 p. m. des 5. Januar in $\mathbf{3} \%$ Robrzucker $+\mathbf{0 , 3} \% \mathrm{NaCl}$ übertragen. - Un 6.35 p. m. des 5 . Januar 
kurze Zehenmuskeln alle wieder ebensogut contrahirbar wie vor Anfang des Versuchs, die übrigen Fussmuskeln (die ihre Erregbarkeit viel später verloren) erst bei $8-10 \mathrm{~cm}$ reizbar (in normalem Zustande bei $16-17 \mathrm{~cm}$ ). Um 10.40 p. m. des 5 . Januar alle Fussmuskeln ungefähr ebenso erregbar wie vor Anfang des Versuchs.

\section{Versuch 5.}

Um 5.12 p. m. des 28. Norember Hinterfuss einer Rana esculenta in $\mathbf{4}^{0} \%$ Rohrzucker gesetzt. - Um 5.23 p. m., die kurzen Zehenmuskeln mit Ausnahme derjenigen der mittleren Zehe schon unerregbar. - Um $5.30 \mathrm{p.m}$. auch die kurzen Zehenmuskeln der mittleren Zehe unerregbar; die übrigen Fussmuskeln contrahiren sich noch lebbaft. - Um 10.23 p. m. des 28 . November alle Fussmuskeln mit dusnahme der kurzen Zehenmuskeln noch etwas erregbar. - Um 9.20 a. m. des 29. November sämmtliche Fussmuskeln unerregbar. Darauf so viel festes $\mathrm{NaCl}$ zugesetzt, dass die partielle Concentration des $\mathrm{NaCl} \mathbf{0 , 1 5} \%$ beträgt. - Um 10.10 a. m. kurze Zehenmuskeln wieder erregbar, aber Contraction noch ziemlich schwach, die übrigen Fussmuskeln noch nicht deutlich reizbar. - Um 11.32 a. m. kurze Zehenmuskeln fast so gut contrahirbar wie unter normalen Zuständen, die übrigen Fussmuskeln erst ziemlich schwach contrahiriar. Versuch abgebrochen.

$\mathrm{Zu}$ diesen Versuchen ist nur noch zu bemerken, dass Sartorien, die so lange, wie im Versuch 3 , in unerregbarem Zustande gehalten werden, nur selten auf Zusatz von $\mathrm{NaCl}$ sich erholen, während Gastrocnemien und besonders die Fussmuskeln selbst nach bedeutend längerer Zeit sich erholen können. Sarterien gehören überhaupt zu den empfindlicheren Muskeln; es ist bei solchen Versuchen wahrscheinlich die plötzliche Wasserentziehung bei dem Zusatz des Kochsalzes, die sie schädigt. Hat der unerregbare Zustand nur 1-2 Stunden gedauert, so erholen sich auch die Sartorien regelmässig (wenigstens bei Herbstfröschen), namentlich wenn der Kochsalzzusatz nur allıählich geschieht.

Die angeführten Versuche sprechen zwar ausserordentlich stark zu Gunsten der Annahme, dass die Unerregbarkeit der Muskeln in reinen Rohrzuckerlösungen bloss eine Folge der Fntziehung von Natriumchlorid ist, und dass der Rohrzucker nur eine passive Rolle spielt; sie schliessen indessen die Möglichkeit nicht völlig aus, dass der Rohrzucker an und für sich schädlich wirkt, dass aber diese Wirkung durch $\mathrm{NaCl}$ anfgehoben wird. In der nächsten Mittheilung werden wir in der That über solche antagonistische Wirkungen gewisser Verbindungen auf die Muskeln und Nerven zu berichten haben. Die folgenden Versuche, in denen Rohrzucker durch 
isosmotische Lösungen von anderen organischen Verbindungen ersetzt ist, beweisen indessen, dass wir es in dem vorliegenden Falle nicht mit solchen antagonistischen Wirkungen zu thun haben.

\section{Versuch 6.}

Un 7.25 p. m. des 19. Juni 1901 wurde ein Sartorins von Rana frnsca, der nach 2 stündigem Liegen in $0,6 \% \mathrm{NaCl} 13 \mathrm{cg}$ wog, in $3,5 \%$ Traubenzucker übergeführt. - Um 8.05 p. m. des 19. Juni keine Erregbarkeit melur; Aussehen des Muskels wie im normalen Zustande, nur etwas weniger durchscheinend und nicht ganz so plastisch. - Um $10.18 \mathrm{p}$. m. des 19. Juni so viel festes $\mathrm{NaCl}$ der Lösung zugesetzt, dass die partielle Concentration des $\mathrm{NaCl} 0,13 \%$ beträgt. Um 1.15 a. m. des 20 . Juni $12 \mathrm{cg}$; bei $9 \mathrm{~cm}$ Rollenabstand Contraction deutlich, bei $7-8 \mathrm{~cm}$ recht lebhaft. - Um 11.58 a. m. des 20 . Juni $12^{1 / 4} \mathrm{cg}$; bei $5 \mathrm{~cm}$ Rollenabstand reizbar, aber Contraction nur mässig stark.

\section{Versuch 7 .}

Um 7.45 p. m. des 19. Juni 1901 Gastrocnemius + Nerv, die nach 21/4 Stunden Liegen in $0,6^{\circ}{ }_{0} \mathrm{NaCl} 78 \mathrm{cg}$ wogen (Gewicht noch in Zunahme begriffen), in $3,5 \%$ Traubenzucker übertragen. In den ersten Minuten nach der Uebertragung häufige und ziemlich starke Zuckungen. Vom Nerven aus in Anfang des Versuchs bei $18 \mathrm{~cm}$ reizbar. - Um 9.30 p. m. Contraction bei directer Reizung des Muskels nur noch sehr schwach, vom Nerven aus keine Contraction zu erzielen. - Um 10.15 p. m. 78 cg; vom Nerven ans unreizbar, Nuskel direct nur noch an einer Stelle reizbar bei völlig genäherten Rollen. - Um $10.18 \mathrm{p} . \mathrm{m}$. so viel festes $\mathrm{NaCl}$ zugesetzt, als einer partiellen Concentration des $\mathrm{NaCl}$ von $0,13 \%$ entspricht. - Um 11.50 a. m. des $20 \mathrm{Juni} 74 \mathrm{cg}$; direct bei $5-6 \mathrm{~cm}$ reizbar, vom Nerven aus unreizbar.

\section{Versuch s.}

Um 10.42 p. m. des 27. April 1902 Hinterfuss einer Rana esculenta, dessen kurzen Zehenmuskeln nach längerem Liegen in $0,7 \% \mathrm{NaCl}$ bei $8-10 \mathrm{~cm}$ contrahirbar waren (die übrigen Fussmuskeln bei $16-17 \mathrm{~cm}$ ), in $3,2 \%$ Mannit recryst. übergeführt. - Um 7.58 a. m. des 28 . April alle kurzen Zehenmuskeln völlig unerregbar, die ïbrigen sehr schwer reizbar. - Um $2.05 \mathrm{p}$. m. diese letzten Muskeln nur noch eben merklich reizbar bei völlig genäherten Rollen. Darauf so viel festes $\mathrm{NaCl}$ hinzugefügt, dass eine $0,1 \%$ ige $\mathrm{NaCl}$-Lösung entstehen musste. Um 7.35 p. m. kurze Zehenmuskeln bei $7 \mathrm{~cm}$ Rollenabstand, die übrigen Fussmuskeln bei $15-16 \mathrm{~cm}$ reizbar, Contraction ziemlich ergiebig, aber träger als normal.

\section{Tersuch 9.}

Un 7.06 p. m. des 30 . April 1902 Hinterfuss einer Rana esculenta in 1,74\% A lanin (isosmotisch mit 6\% Rohrzucker) gesetzt. Da die Lösung eben merklich sauer reagirte, wurde 1: $7500 \mathrm{Na}_{2} \mathrm{CO}_{3}$ zugesetzt, was die Reaction in eine sehr schwach alkalische umwandelte. - Um $9.15 \mathrm{p}$. m. des 30 . April alle kurzen Zehenmuskelı völlig unerregbar, die andern Fussmuskeln bei $10-12 \mathrm{~cm}$ (statt 
16-17 cm) reizbar und Contraction etwas träge. - Um 12.45 p. m. des 1. Mai alle Fussmuskeln unerregbar. - Um 12,50 p. m. so viel festes $\mathrm{NaCl}$ zugesetzt, als einer $0,1 \%$ igen Lösung entspricht. - Um 5.00 p. m. des 1 . Mai kurze Zehenmuskeln bei $5-6 \mathrm{~cm}$ (vor Anfang des Versuchs bei $9-10 \mathrm{~cm}$ ) Rollenabstand, die übrigen Fussmuskeln bei $10-12 \mathrm{~cm}$ reizbar, Contraction etwas schwach. Um 10.07 p. m. des 1. Mai Erregbarkeit ungefähr gleich geblieben. - Um 10.00 p. m. des 2. Mai alle Muskeln noch erregbar.

In ganz älnlicher Weise werden Muskeln nach einiger Zeit in Lösungen von Milchzucker, Erythrit, Taurin und Aspa$\mathrm{l}^{\prime} \mathrm{ag}$ in, die mit $5-6^{0}{ }_{0}$ igen Rohrzuckerlösungen isosmotisch sind, völlig unerregbar. Die Asparaginlösungen werden am besten mit $\mathrm{MgO}$ neutralisirt, da die zur Nentralisation erforderliche Menge von $\mathrm{Na}_{2} \mathrm{CO}_{3}$ das Eintreten des unerregharen Zustandes stark verzögert. Taurinlösungen reagiren kaum merklich saner, und die Spur $\mathrm{Na}_{2} \mathrm{CO}_{3}$, die erforderlich ist, um die Lösung völlig neutral oder eben merklich alkalisch zu machen, kommt nicht in Betracht.

In einheitlichen Lösungen aller jener Verbindungen, die rasch in die lebenden Muskelfasern eindringen, wozu, wie in der ersten dieser Mittheilungen gezeigt worden ist, die grosse Mehrzahl der organischen Verbindungen gehört, erfolgt natürlich vollständige Wasserstarre der Muskeln, ehe ein unerregbarer Zustand der Muskeln in Folge des Entzuges von Chlornatrium eintreten kann. Bei Benutzung dünner Muskeln, etwa des M. cut. pectoris oder der kurzen Zehenmuskeln (auch des Sartorius) lässt sich dagegen leicht nachweisen, dass in den Lösungen von langsamer eindringenden Verbindungen, wie Harnst off und Glycerin (schwieriger bei Glykol und gleich schnell durchgehenden Verbindungen) die Abwesenheit von Chlornatrium viel früher Unerregbarkeit der Muskeln veranlasst, als der allmählichen Wasseraufnahme der Muskeln entspricht, $d . h$. dass diese Unerregbarkeit in der ersten Zeit nicht als Wasserstarre zu deuten ist, sondern wirklich der Exosmose des Chlornatriums zuzuschreiben ist. Durch Wägungsversuche kann dieser Schluss noch sicherer gestellt werden. In zusammengesetzten Lösungen, wie etwa $6 \%$ Rohrzucker $+2 \%$ Aethylalkohol, $1,7 \%$ Alanin $+1: \%$ Aceton etc., in denen Wasserstarre nicht eintreten kann, werden Muskeln ebenfalls unerregbar, um bei Zusatz von $0,1-0,15 \%_{0}$ Chlornatrium zu diesen Lösungen ihr Contractionsvermögen wieder zu gewinnen.

Aus allen diesen Versuchen geht mit Gewissheit (d. h. mit überwältigender Wahrscheinlichkeit) hervor, 
dass beim Verweilen von Muskeln in 6\%igen Rohrzuckerlösungen oder in mit diesen isosmotischen Lösungen von Traubenzucker, Fructose, Milchzucker, Mannit, Alanin etc. der bald eintretende unerregbare Zustand nur durch die Verarmung der Zwischenflüssigkeit der Muskeln an Chlornatrium bedingt sein kann, indem letzteres a us den Muskeln in die Rohrzuckerlösung etc. exosmirt.

Dass eine solche Exosmose des Natriumchlorids aus der Zwischenflüssigkeit der Muskeln wirklich stattfindet, kann übrigens sehr leicht uachgewiesen werdeı durch den folgenden Versuch: Etwas grössere Muskeln, z. B. der Gastrocnemius oder Gracilis + Semimembranosus werden zunächst in einer $6 \%$ igen Rohrzuckerlösung abgespült und eventuell einige Minuten in einer solchen Lösung gelassen, um die Muskeln von der oberflächlich adhärirenden Lymphe oder Kochsalzlösung zu befreien. Darauf werden die Muskeln in eine frische Lösung von $0^{2} \%$ Rohrzucker gesetzt, wobei das Volumen der Lösung im Vergleich zu dem Volumen der angewandten Muskeln nicht zu gross sein darf. Nachdem die Muskeln in dieser Lösung längere Zeit verweilt haben, tritt auf Zusatz von angesäuertem Silbernitrat zu der Lösung ein Niederschlag auf, dessen weitere Identificirung als AgCl keine Schwierigkeiten bereitet. Da wir annehmen dürfen, dass die Salze der Zwischenflüssigkeit der Muskeln im Wesentlichen dieselbe Zusammensetzung haben wie im Blutplasma des Frosches, also ganz vorwiegend aus $\mathrm{NaCl}$ bestehen, und die Zwischentlüssigkeit daher, von seinem Gehalt an organischen Verbindungen abgesehen, im Wesentlichen eine $0,6 \%$ ige $\mathrm{NaCl}$ darstellt, so wäre es sogar im Principe möglich, das Volumen der Zwischenflüssigkeit der Muskeln aus der Menge des exosmirenden Natriumchlorids zu bestimmen.

Eine quantitative Bestimmung der Salze von frischen Froschmuskeln, deren Blutgefässe vor der Verascherung nicht ausgespült waren, ist von Katz ausgeführt worden. Seine Daten mögen hier mitgetheilt werden.

Auf 1000 Th. friseher Muskeln kommen:

\begin{tabular}{lcccccccc} 
& $\mathrm{K}$ & $\mathrm{Na}$ & $\mathrm{Fe}$ & $\mathrm{Ca}$ & $\mathrm{Mg}$ & $\mathrm{P}$ in toto & $\mathrm{CI}$ & $\mathrm{S}$ \\
Versuch I & 3,0966 & 0,5561 & 0,0662 & 0,1557 & 0,2419 & 1,8078 & 0,4057 & 1,6152 \\
Versuch II & 3,0627 & 0,5485 & 0,0584 & 0,1575 & 0,2287 & 1,9161 & 0,3903 & 1,6507 \\
\hline Nittel & 3,0797 & 0,5523 & 0,0629 & 0,1566 & 0,2353 & 1,8620 & 0,4025 & 1,6330
\end{tabular}


Der Schwefel stammt fast ausschliesslich von der Verbrennung der Muskelproteine her. Der weitaus grösste Theil des Phosphors (1,4952 resp. 1,5512, im Mittel 1,5232) befand sich in dem wässerigen Auszug des Muskels und war zweifellos als Phosphat im Muskel enthalten. 0,1931 resp. 0,2209 (im Mittel 0,2070) Theile Phosphor fanden sich im alkoholisehen Auszuge der Muskeln und werden vorwiegend aus dem Lecithin des Muskels stammen. Rechnet man den Phosphor des wässerigen Auszuges in dem Gehalt der Muskeln an $\mathrm{PO}_{4} \mathrm{H}$ um, so erhält man die Werthe $4,635,4,808$ und (Mittelwerth) 4,722.

Auch aus diesen Daten würde sich ein maximaler Werth für das Volumen des Blutplasmas + der Zwischenflüssigkeit der Muskeln im Verhältniss zu dem Volumen der Muskelfasern + Bindegewebszellen ausrechnen lassen, indem man die $Z$ wischenflüssigkeit als eine $0,6 \%$ ige $\mathrm{NaCl}$ betrachtet und den ganzen Chlorgehalt der Muskeln dieser Zwischenflüssigkeit und dem Blutplasma zuschreibt.

\section{Wird durch die Abwesenheit von Chlornatrium in der Lösung zwischen den Muskelfasern bloss das Contractionsvermögen des Iuskels aufgehoben oder zugleich die Erregungsleitung durch seine Substanz unmöglich gemacht?}

Biedermann zeigte vor längerer Zeit, dass Muskeln unter Umständen ihr Contractionsvermögen verlieren können, ohne dass die Erregungsleitung durch ihre Substanz anfgehoben wird (vgl. auch die erste dieser Mittheilungen). Die Frage, ob bei Entziehung des $\mathrm{NaCl}$ aus der Zwischenfüssigkeit der Muskeln das Vermögen, Erregungen zu leiten, nicht vielleicht bestehen bleibt, liegt auch um so näher, als wie schon bei der Besprechung der Vorversuche erwähnt, die Nerven ihre Erregbarkeit und Erregungsleitung in reinen Zuckerlösungen thatsächlich beibehalten.

Die folgenden Versuche entscheiden diese Frage in negativem Sinne; bei der Entzichung vom Chlornatrium wird auch das Vermögen der Muskeln, Erregungen zu leiten, völlig aufgehoben, um sich bei Kochsalzzufuhr wieder einzustellen.

Versuch 10.

Un 4.00 p. m. des 23. Juli 1901 wurde ein Sartorius einer grossen Rana esculenta, der nach 1 stïndjgem Liegen in $0,6 \% 0 \mathrm{NaCl} 38 \mathrm{cg}$ wog, so aufgehängt, dass seine proximale Hälfte in eine $6^{\circ} \%$ ige Rohrzuckerlösung untertauchte, seine 
distale Hälfte dagegen sich in einem dampfgesättigten Raum oberhalb der Lösung befand. - Um 5.15 p. m. contrahirt sich die distale Hälfte des Muskels ziemlich stark schon bei einem Rollenabstand von $12 \mathrm{~cm}$, wenn die Reizelektroden auf sie direct angelegt werden, gar nicht, wenn die Elektroden auf die proximale Hälfte des Muskels angebracht werden; nur bei fast völlig genäherten Rollen findet in letzterem Falle, zweifellos in Folge von Stromschleifen, Contraction der distalen Hälfte des Muskels statt. Die proximale Hälfte bei jeder Lage der Reizelektroden uncontrahirbar. Die Reizungsversuche wurden mehrmals wiederholt, stets mit gleichem Resultat. - Un 6.23 p. m. des 21 . Juli das proximale Ende anf etwa $1 / 8$ Länge des Muskels in $4 \%$ Rohrzucker $+0,2 \% \mathrm{NaCl}$ getaucht. - Um 7.25 p. m. contrahirt sich jedes Ende des Muskels allein für sich, wemn die Reizelektroden auf das eine oder das andere Muskelende angelegt werden, nur bei sehr grosser Rollenanwäherung geht die Erregung scheinbar durch das Mittelstück des Muskels hindurch auf das andere Ende, so dass beide Enden sich contrahiren, während das Mittelstück in Ruhe bleibt. Auch in diesem Falle handelt es sich zweifellos um Stromschleifenbildung. Durch einen Zufall gerieth später die ganze proximale Hälfte des Muskels in die Lösung von $4 \%$ Rohrzucker $+0,2 \% \mathrm{NaCl}$ und gewann dadurch sein Contractionsvermögen wieder. - Um. 9.50 p. m. die ganze proximale Hälfte des Muskels auf's Nene in reine $6 \%$ oige Rohrzuckerlösung eingetaucht. - Um $11.25 \mathrm{p} . \mathrm{m}$. wurde der in der Zuckerlösung befindliche Abschnitt des Muskels in der Mitte durch einen Seidenfaden stark eingeschnürt, ohne dass die geringste Zuckung in der einen oder der anderen Hälfte des Muskels erfolgte. - Um 11.33 p. m. der ganze Iuskel in $0,6 \%$ NaCl übergeführt. - Um 11.58 p. m. reagiren beide Enden des Muskels sowohl auf elektrischer wie auf mechanischer Reizung, und zwar so, dass die Reizung des einen Endes eine Zuckung in dem ganzen Muskel auslöst. Am nächsten Morgen das distale Ende des Muskels noch gut erregbar, das proximale Ende todt.

\section{Versuch 11.}

Um 5.52 p. m. des 31. Juli 1901 wurde der Sartorius einer Rana esculenta $V$-förmiğ umgebogen und nur mit dem mittleren Theile in $6^{\circ}$ ige Rohrzuckerlösung gesetzt, während die beiden Endeu des Muskels sich in dem Dampfraum oberhalb der Lösung befanden. - Um 9.40 p. m. an beiden Enden gut erregbar, die Erregung aber selbst bei röllig genäherten Rollen nicht durchgeleitet, so dass sich stets nur das gereizte Ende contrahirt. Darauf wurde der ganze Muskel in $0,6 \% \mathrm{NaCl}$ zurückgesetzt. - Um $10.30 \mathrm{p}$. m. contrahirt sich der Muskel vorzüglich schon bei $10--11 \mathrm{~cm}$ Rollenabstand in seiner ganzen Länge, wenn er an dem einen oder dem andern Fnde gereizt wird. - Um 10.31 p. m. der mittlere Theil wieder in die $6 \%$ ige Zuckerlösung gesetzt. - Un $9.45 \mathrm{a} . \mathrm{m}$. des 1. Augnst beide Enden, jedes für sich, gut erregbar; die Erregung pflanzt sich aber $\mathrm{n}$ ich $\mathrm{t}$ fort durch das Mittelstück, gleichgültig ob das eine oder andere Ende elektrisch oder mechanisch gereizt wird. Darauf der ganze Muskel noch einmal in $0,6 \% \mathrm{NaCl}$ übertragen. - Um 10.14 a. m. zuckt der ganze Muskel, wenn er an dem einen Ende durch eine Ligatur zum Theil durchschnürt wird; die Contraction ist aber nur noch schwach, was indessen zweifellos nur eine Folge der mehrfachen Misshandlungen ist. 
Eine Anzahl ähnlicher Versuche, die in Details verschiedenartig. modificirt wurden, ergab stets dieselben Resultate.

Namentlich aus Versuch 11 ergibt sich mit völliger Sicherheit, dass es sich bei den kochsalzfreien Muskeln nicht etwa bloss um den Verlust der Fähigkeit zur Reizaufnahme (Reizperception) und des Contractionsvermögens, sondern thatsächlich zugleich um eine Aufhebung der Fähigkeit, Erregungen fortzupflanzen, handelt, denn in diesem Versuche hat der Muskel den Reiz that sächlich percipirt, wie aus der Contraction des unmittelbar gereizten Endes zu ersehen ist, während die Erregung nicht durch das salzfreie (genauer salzarme) Mittelstück auf das andere, ebenfalls für sich contractionsfähige Ende des Muskels übergeht.

Bei kieineren Muskeln ist die mechanische Reizung (am sichersten durch starke Ligatur) vielfach zweckmässiger als die elektrische, da bei der ersten Reizungsart Irrthümer durch Stromschleifenbildung veranlasst werden können. Es ist stets geboten, durch die nachträgliche Ueberfübrung des unerregbaren Muskelabschnitts in eine Kochsalzlösung sich davon zu überzeugen, dass dieser Abschnitt wirklich nur vorübergehend unerregbar war, nicht getödtet worden ist. Selbst in Folge einer partiellen Durchschnürung des Muskels durch einen Faden werden die betreffenden Muskelfasern zunächst nur in unmittelbarer Nähe der Ligatur getödtet; das fortschreitende Absterben der Fasern nach beiden Seiten von der Ligatur geht sehr langsam vor sich, so dass das Kochsalz (von 0,2\% $\%$ igen Lösungen an) reichlich Zeit findet, in die Zwischenflüssigkeit der Muskeln an dem betreffenden Muskelabschnitt in genügenden Mengen zu diffundiren, ehe die Erregbarkeit dieses Absehnittes durch den Tod endgültig aufgehoben worden ist.

Ehe ich diesen Abschnitt schliesse, möchte ich für einen Augenblick auf die Verhältnisse bei den Nerven zurückkommen. Es wurde bei Besprechung der Vorversuche angegeben, dass, wenn bei einem Nerv-Muskelpräparat (Gastrocnemius-Ischiadicus) der Nerv allein in $6-70_{0}$ iger Rohrzuckerlösung gesetzt wird, während der Muskel in einem dampfgesättigten Raum gehalten wird, eine Zuckung des Muskels vom centralen Ende des Nerven aus selbst nach 20 und mehr Stunden ausgelöst werden kam - dass überhaupt die Erregbarkeit des Nerven in keiner Weise leidet. Diese Versuche beweisen indessen nicht ohne Weiteres, dass bei der Erregungsleitung durch die Nerven die Anwesenheit eines Natriumsalzes in der Nachbarschaft 
der Axencylinder entbehrlich ist. Diese Angelegenheit steht nämlich im Zusammenhange mit der noch nicht erledigten Frage nach den morphologischen und physiologischen Beziehungen zwischen dem Axencylinder und dem Nervenmark. Nach Ranvier würde jedes Marksegment zusammen mit der Schwann'schen Scheide zwischen je zwei Schnürringen (Anneaux constricteurs) oder Ranvier'schen Einschnürungen, wie sie jetzt vielfach benannt werden, eine Zelle darstellen. Die eigentliche Marksubstanz (Myelin) ist nach Ranvier"s Annahme von einer dünnen Protoplasmalage allseitig unschlossen und wäre also etwa mit dem vom Primordialschlauch eingeschlossenen Zellsafte einer Pflanzenzelle zu vergleichen. Diese Zellen würden stark verbreitete Ringe oder Reifen ${ }^{1}$ ) darstellen, durch welche die Axencylinder, die als Fortsätze ron Ganglienzellen (Neuronen) aufgefasst werden müssen, hindurchlaufen wie in einem Tunnelsystem. Wenn nun zwischen dem Axencylinder und der inneren Fläche dieser Markscheiden ein periaxialer Lymphr a $\mathrm{m}$ besteht, wie Schiefferdecker annimmt, und wie man folgerichtig annehmen muss, wenn die Ranvier'sche Ansicht richtig ist, so wäre die Erbaltung der Erregbarkeit von Nerven in reinen Zuckerlösungen mit dem Postulat vereinbar, dass zur Erregungsleitung die Anwesenheit eines Natriumsalzes in der Umgebung der Axencylinder nöthig ist. Die einzelnen Segmente des Nervenmarkes haben nämlich beim Frosche eine Länge von 1,5-1,8 mm, und da es sicher ist, dass Kochsalz ${ }^{2}$ ) nicht durch die Marksubstanz hindurch diffundiren kann, so würde die Exosmose des Natriumchlorids aus dem periaxialen Lymphraume nur an Stelle der Ranvier'schen Ringe

1) Zellen in der Form von Ringen oder Reifen kommen auch im Pflanzenreich ein Mal vor, nämlich in den Antheridien der Farnprothallien, wo zwei solche über einander stehende Ringzellen die Seitenwantung der Antheridien bilden und die Spermatozoiden bildenden Zellen umschliessen.

2) Ranvier zeigte, dass Carminpräparate, Silbernitrat etc. nur an Stelle der Schurringe in die Nervenfasern eindringen, und dass die Färbung sich von hier ans verbreitet. Er verallgemeinerte diese Erfahrung dahin, dass auch die Nährstoffe nur von diesen Stellen aus aufgenommen werden können, was für die Zuckerarten, die meisten Salze etc. zweifellos richtig sein wird. Alle oder wenigstens die meisten jener Verbindungen, die nach der ersten dieser Mittheilungen in die rerschiedensten lebenden Gewebezellen sehr schnell eindringen, können dagegen direct durch die Marksubstanz wandern, wie durch Studien über die zeitlichen Verhältnisse bei der Narkose der Nervenstämme festgestellt werden kann. 
geschehen können. Bei der ausserordentlich geringen Weite der ringförmigen Spalten, von denen die Diffusionsströme ausgehen würden, die nur einen Bruchtheil eines $\mu$ betragen kann, dürfte es mehrere Tage dauern, bis die Kochsalzconcentration in dem periaxialen Raume nur auf den halben Werth sinkt. Eine sichere Entscheidung der Frage, ob eine Erregungsleitung durch die Nerven ohne die Gegenwart eines Natriumsalzes in der Umgebung der Nervenfasern resp. der Axencylinder möglich ist, dürfte nur durch Versuche mit marklosen Nerven zu treffen sein.

3. Wie hoch ist die niedrigste Concentration des Natriumchlorids in der Zwischenflüssigkeit der Yuskeln, die zu einer Contraction des IInskels eben ausreicht?

Die Beantwortung der Frage, die am Kopfe dieses Abschnittes steht, ist in mehr als einer Hinsicht von Bedeutung. In der Finleitung $\mathrm{zu}$ dieser Arbeit wurde angegeben, dass ich die Unerregbarkeit von Muskeln, die in reinen Zuckerlösungen verweilt haben, ursprünglich so erklären wollte, dass in Folge der Exosmose der Elektrolyten aus der Zwischenflüssigkeit der elektrische Widerstand der Muskeln sehr stark zu-, die Stromintensität also sehr stark abnimmt. Es wurde auch erwäbnt, dass gleich bei der Entstehung dieser Hypothese gewisse Bedenken gegen dieselbe sich einstellten. Ein sehr ernstes Bedenken, dem ich nicht auf die Dauer hätte widerstehen können, entsprang daraus, dass der unerregbare Zustand der Muskeln relativ schnell eintritt. Ein Sartorius z. B. wird je nach seiner Grösse in einer reinen $6 \%$ igen Rohrzuckerlösung schon nach 25-51) Minuten völlig unerregbar. Es schien mir nun sehr unwahrscheinlich, dass schon nach dieser kurzen Zeit eine fast vollständige Exosmose der Elektrolyten aus der Zwischenflüssigkeit sich vollziehen könnte. Dies wird aber von der Hypothese gefordert, wie die folgende Ueberlegung zeigt. Selbst bei der Annahme, dass die elektrische Leitfähigkeit des Muskels ausschliesslich in seiner Zwischenflüssigkeit ihren Sitz hat, so würde ein Abfall der Concentrationshöhe der Elektrolyten dieser Zwischenflüssigkeit auf einen Zehntel ihres ursprünglichen Werthes den elektrischen Widerstand des Muskels nur um das Zehnfache oder eigentlich um etwas weniger als das Zehnfache (da bei der Verdünnung der Lösung die Ionisation noch etwas zunimmt) vergrössern. Damit der Widerstand den 
Hundertfachen des ursprünglichen Werthes annimmt und die Stromintensität dementsprechend hundert Mal kleiner wird, müsste die Concentration der Elektrolyten in der Zwischenflüssigkeit mehr als hundert Mal geringer sein als im normalen Zustande ${ }^{1}$ ). Da aber Inductionsströme, die 200- $400 \mathrm{Mal}$ stärker sind, als zur Auslösung einer Zuckung bei einem normalen Muskel ausreicht, oder vielmehr 200-400 Mal stärker sein würden, wenn der Widerstand der Muskeln in beiden Fällen ausgeschaltet wäre, noch immer keine Contraction von Sartorien, die etwa eine Stunde in einer $6 \%$ igen Rohrzuckerlösung verweilt haben, bewirken, so müsste die Auslaugung der Salze aus der Zwischenflussigkeit der Muskeln innerhalb dieser relativ kurzen Zeit eine noch vollständigere sein, wenn die ursprüngliche Hypothese des unerregbaren Zustandes richtig gewesen wäre. Eine so weitgehende Auslaugung in so kurzer Zeit musste von vorn herein fast unmöglich erscheinen und stimmte namentlich sehr schlecht zu den Vorstellungen über die Geschwindigkeit der Diffusion von Salzen durch die Zwischenflüssigkeit, die ich aus Wägungsversuchen gewonnen hatte. Wenn z. B. ein Sartorius aus einer $0,7^{0}{ }_{0}$ igen in eine $0,4^{\circ} \%$ ige Chlornatriumlösung versetzt wird, vergeben 2-3 Stunden, bis wieder eine praktische Gewichtsconstanz des Muskels eingetreten ist, während andererseits die Ueberführung eines Sartorius aus einer $0,41 \%$ igen in eine $0,40 \%$ ige Kochsal rlösung eine so geringe Gewichtszunahme verursacht, dass dieselbe kaum noch experimentell direct nachgewiesen werden kann. Freilich sind bei solchen Versuchen zwei verschiedene Vorgänge im Spiele, die Exosmose des Natriumchlorids aus dem Muskel und das Einwandern von Wasser in die Muskeln; doch ist nicht wahrscheinlich, dass der letztere Vorgang, der natürlich die eigentliche Ursache der Gewichtszunahme ist, dem ersteren Vorgang stärker nachlinkt.

Wenn sich nun nachweisen liesse, dass es eine minimale Chlornatrium-Concentration gibt, unterhalb welcher noch

1) Dies würde nur strenge richtig unter der Voraussetzung sein, dass beim Verweilen der Muskeln in den Zuckerlösungen die Gestalt und das Volumen der Räume zwischen den Muskelfasern unverändert bleiben, was namentlich für Rohrzuckerlösungen nicht völlig zutrifft. Bei Berechnung der relativen Stromstärken in den Muskeln ist die bei Inductionströmen nicht ganz richtige Annahme gemacht worden, dass der Widerstand des Stromkreises ausserhalb der Muskeln zu klein ist, um in Betracht zu kommen. 
so starke Inductionsströme keine Contraction des Muskels mehr hewirken, so würde man einerseits einen festen Ausgangspunkt gewinnen, um die mehrfach erwähnte Hypothese in strenger Weise durch quantitative Betrachtungen ${ }^{1}$ ) zu widerlegen, und andererseits eine Methode, um die Geschwindigkeit der Exosmose ron Chlornatrium aus den Muskeln zu bestimmen.

Bei dünneren Muskeln, wie dem M. cutaneus pectoris, den kurzen Zehenmuskeln, den Sartorien erwacbsener Amphibien und bei den übrigen quergestreiften Muskeln möglichst kleiner Thiere (z. B. von Fröschen währesd und kurz nach der Metamorphose), lässt sich nun in der That eine solche minimale Concentration nit bedeutender Schärfe leicht feststellen.

$\mathrm{Zu}$ dem Zrecke werden die Muskeln zunächst in Lösungen, die neben $6 \%$ Rohrzucker noch $0,05,0,06,0,0 ;$ oder $0,08 \%$ Chlornatrium enthalten, gesetzt. In den beiden ersten Lösungen (nit 0,05 , resp. $0,06 \% \mathrm{NaCl}$ ) werden die Muskeln stets nach einigen Stunden unerregbar; in einer Lösung, die neben $6 \%$ Rohrzucker noch $0,08 \%$ Chlornatrium enthält, verlieren dagegen Muskeln ihre Erregbarkeit kaum früher als unter normalen Umständen; die Contraction solcher Muskeln ist indessen noch eine recht schwache. In $6^{0}: 0$ Rohrzucker $+0,0 ; \% \mathrm{NaCl}$ waren die Ergebnisse etwas unsicher, bisweilen konnte eine eben merkliche Contraction der Muskeln in dieser Lösung noch nach 24 Stunden wahroenommen werden, bisweilen schienen die Muskeln (namentlich der M. cut. pectoris) ihre Erregbarkeit in derselben nach 10-12 Stunden röllig eingebüsst zu haben.

Durch diese Versuche wurde jedenfalls festgestellt, dass die minimale Concentration des Chlornatriums, unterhalb der keine Contraction mehr stattfinden kann, höher als $0,06 \%$ liegt, denn es rersteht sich von selbst, dass, sobald die Concentration des $\mathrm{NaCl}$ in der Zwischenflüssigkeit der Muskeln auf $0,06^{\circ}: 0$ gesunken ist, kein Natriumchlorid mehr in eine den Muskel umgebende Lösung diffundiren lann, die ebenfalls $0,00^{\circ}: 0 \mathrm{NaCl}$ enthält (die minimale Aenderung der Chlornatriumconcentration, welche dem Gleichgewichtszustand entspricht, die durch die Gegenwart von Eiweiss in der Zwischen-

1) Eine directe experimentelle Bestimmung des elektıischen Widerstands ron Muskeln, deren Zwischenflissigkeit durch isosmotische Lösungen von reinem Zucker oder von isosmotischen Gemischen ron Zucker und Chlornatrium in bekannten Verhältnissen ersetzt ist, hofie ich demnächt auszufúhren. 
flüssigkeit der Muskeln bedingt wird, kann hier füglich vernachlässigt werden). Indem man nun Muskeln, die in Lösungen vou $6 \%$ Rohrzucker $+0,05-0,06 \% \mathrm{NaCl}$ schon völlig unerregbar geworden sind, in Lösungen von $6 \%$ Rohrzucker + $0,08 \% \mathrm{NaCl}$ überführt, bemerkt man, dass sie nach längerer Zeit wieder erregbar werden. Durch eine grössere Anzahl Versuche dieser Art konnte ich die minimale Concentration des $\mathrm{NaCl}$, bei welcher Erregbarkeit noch besteht, zwisehen den beiden Grenzwerthen 0,068 und 0,0 $4 \%$ einschliessen. Dies gilt speciell für die Sartorien und die kurzen Zehenmuskeln; bei dem Gastrocnemius und anderen dickeren Muskeln liegt die minimale Concentration des $\mathrm{NaCl}$ sicher zwischen $\mathbf{0 , 0 6}$ und $0.08 \%$. Wegen der Langsamkeit der Diffusion ist es schwer die minimale Concentration hier noch genauer festzustellen. Bemerkenswerth ist die relativ lange Erhaltung der Erregbarkeit der Hinterfussmuskeln (mit Ausnahme der kurzen Zehenmuskeln) in reinen Zuckerlösungen oder in Zuckerlösungen, die nur noch 0,04-0,05\% $\mathrm{NaCl}$ enthalten. Es scheint dies daher zu kommen, dass die Fussknochen ihr $\mathrm{NaCl}$ recht langsam abgeben, und da dieses Chlornatriurn durch die Muskeln hindurch gehen muss, so bleibt der Natriumgehalt dieser Muskeln lange Zeit etwas über der minimalen Concentration.

Bis die Concentration des Chlornatrinms in der Zwischenflüssigkeit der Muskeln unter $0,15 \%$ gesunken ist, kann man wenigstens ohne graphische Hülfsmethoden kaum einen Unterschied in der Erregbarkeit und Contractionsstärke gegenüber normalen Muskeln wahrnehmen. Auch bei einem Gehalt der Zuckerlösung von $0,12 \%$ $\mathrm{NaCl}$ ist die Erregbarkeit nach dem Eintritt von Gleichgewicht nur sehr wenig herabgesetzt. Bei einem Gehalte von nur $0,1 \%$ ist nach dem Diffusionsausgleich die Erregbarkeit sehr deutlich vermindert und fällt dann bei der weiteren Concentrationsabnahme des $\mathrm{NaCl}$ sehr schnell herab, bis sie bei circa $0,07 \%$ auf Null sinkt.

Dank dieser sehr schnellen Abnahme in der Erregbarkeit von Muskeln, während das Chlornatrium-Gehalt ihrer Zwischenflüssigkeit von 0,1 auf $0,07 \%$ sinkt, eine Abnahme, die unmöglich auf dem grösseren elektrischen Widerstand der Muskeln beruhen kann, lässt sich mit Leichtigkeit der Zeitpunkt feststellen, wo die Concentration des $\mathrm{NaCl}$ in der Zwischenflüssigkeit der Muskeln nur noch $0,07-$ $0,08 \%$ beträgt und somit auch die Geschwindigkeit der Exosmose des Natriumchlorids aus den Muskeln bestimmen. Bei der Ueber- 
führung eines M. cut. pectoris aus $0,6 \% \mathrm{NaCl}$ in reine $6 \%$ ige Rohrzuckerlösungen sinkt der $\mathrm{NaCl}$-Gehalt der Zwischenflüssigkeit auf eirea $0,08 \%$ schon nach wenigen Minuten; bei Sartorien ist diese Concentration unter gleichen Umständen je nach ihrer Grösse bei Temperaturen von circa $18^{\circ} \mathrm{C}$. in etwa $30-50$ Minuten erreicht, bei Gastrocnemien von $70-100$ egr Gewicht in circa $3-1$ Stunden. Diese Resultate stimmen sehr gut überein mit den Schätzungen über die Geschwindigkeit der Diffusion von $\mathrm{NaCl}$ in den Muskeln, die aus Wägungsversuchen abgeleitet werden können.

Die Aenderung in der Zuckungsform von Muskeln, deren Zwischenflüssigkeit eine immer weitergebende Verarmung an $\mathrm{NaCl}$ erleidet, wnrde noch nicht eingehender studirt. Im nächsten Herbst hoffe ich über diesen Gegenstand und über die Aenderungen in den mechanischen Eigenschaften solcher Muskeln weitere Studien anzustellen; die gegenwärtige Jahreszeit ist zu einer solchen Untersuchung wenig geeignet.

4. Kanm Natriumchlorid in seiner Eigenschaft, die Erregbarkeit von Muskeln zu erhalten, durch andere Natriumsalze ersetzt werden?

Diese Frage kann nit aller Bestimmtheit in positivem Sinne beantwortet werden, und zwar haben zablreiche Versuche ergeben, dass alle Natriumsalze, deren negativer Bestandtheil nicht eine direct schädigende Wirkung auf die Muskeln ausübt, die Rolle des Natriumchlorids übernehmen kömen. Es wurden z. B. die folgenden Natriumsalze geprüft, und zwar sämmtliche mit positivem Erfolge: Natriumbromid, Natriumitrat, Natriumsulfat, sekundäres Natriumphosphat $\left(\mathrm{Na}_{2} \mathrm{HPO}_{4}\right)$, Natriumbicarbonat $\left(\mathrm{NaHCO}_{3}\right)$, Natriumchlorat $\left(\mathrm{NaClO}_{3}\right)$, Natriumacetat, Natriumbenzoat, Natriumsalicylat, Ferrocyannatrium $\left(\mathrm{Na}_{4} \mathrm{Fe}(\mathrm{CN})_{6}+10 \mathrm{Aq}\right)$ und Ferricyannatrium $\left(\mathrm{Na}_{3} \mathrm{Fe}(\mathrm{CN})_{6}+\right.$ $2 \mathrm{Aq})$.

Um alle Irrthümer bei der Beantwortung der Hauptfrage völlig auszuschliessen, wurden in den ersten Versuchen mit jedem einzelnen dieser Salze die Muskeln zunächst durch Verweilen in reinen $5-6 \%$ igen Lösungen von Rohrzucker oder isosmotiscben Lösungen von Traubenzucker völlig unerregbar gemacht. Nachdem die Muskeln in diesen Lösungen schon zwei Stunden oder länger unerregbar ge- 
blieben waren, wurden sie entweder in eine ungefähr isosmotische Lösung des zu prüfenden Natriumsalzes übergeführt, oder sie kamen in Lösungen von Rohrzucker (resp. Traubenzucker), denen noch eine gewisse Menge des betreffenden Natriumsalzes zugesetzt war, wobei dafür Sorge getragen wurde, dass diese gemischten Lösungen nicht zu stark wasserentziehend wirken konnten. Letztere Methode hat den grossen Vortheil, dass man die (partiellen) Concentrationen der betreffenden Natriumsalze beliebig abstufen kann. Bei ihrer Anwendung lässt sich in ganz ähnlicher Weise wie bei Natriumchlorid die minimale Concentration der einzelnen Natriumsalze ermitteln, die eben ausreicht, um die Erregbarkeit und das Contractionsvermögen der Muskeln wieder herzustellen. Viele Natriumsalze, welche in Concentrationen, die mit $0,6-0,7 \% \mathrm{NaCl}$ isosmotisch sind, einen todtlichen oder entschieden schädlichen Einfluss auf die Muskeln ausüben, besitzen keine solche schädigende Wirkung in niedrigeren Concentrationen - Concentrationen, die aber ihrerseits noch völlig ausreichen, um den Muskeln ihr Concentrationsvermögen wieder zu verleihen. In einer Lösung von $1,5^{\circ} \%$ benzo ësaurem Natrium, die mit $0,6 \% \mathrm{NaCl}$ isosmotisch ist, z. B. sterben Muskeln recht schnell, während in Lösungen, die neben $5-6 \%$ Rohrzucker noch $0,25 \%$ benzoësaures Natrium entbalten, das Contractionsvermögen von Muskeln sich über 24 Stunden erhält. In diesem speziellen Falle ist freilich die Contraction schwächer als in äquivalenten Concentrationen von $\mathrm{NaCl}$. Ganz ähnlich verbält es sich mit chlorsaurem Natrium $\mathrm{NaClO}_{3}$.

Um die minimale Concentration aller genannten Natriumsalze zu bestimmen, die eben ausreicht, um die Muskeln erregbar zu halten, wären sehr zahlreiche und zeitraubende Versuche nothwendig gewesen, wenn ich diese Concentration mit derselben Schärfe wie bei Chlornatrium feststellen wollte. Ich begnügte mich daunit, einerseits das Verhalten von Muskeln zu beobachten, die in Lösungen gesetzt wurden, welche neben $6 \%$ Rohrzucker noch so viel von den einzelnen Natriumsalzen enthielten, als einer $0,05 \%$ igen $\mathrm{NaCl}$ entspricht, andererseits das Verhalten von Muskeln in Lösungen, in denen sich ausser Rohrzucker noch mit $0,1 \% \mathrm{NaCl}$ äquivalente Mengen der einzelsen Natriumsalze befanden, zu untersuchen. In den erstgenannten Lösungen reichte die Natriummenge in keinem einzigen Falle aus, um die Erregbarkeit der Muskeln wieder herzustellen, bezw. um ihre Erregbarkeit zu erhalten. In der zweiten 
Reihe von Versuchen dagegen wurde die Erregbarkeit regelmässig wieder hergestellt, respective erhalten und in den meisten Fällen war die Erregbarkeit und die Contractionsstärke, namentlich in den ersten Stunden, fast genau dieselbe wie in Lösungen von $6 \%$ Robrzucker $+0,1^{0_{0}} 0 \mathrm{NaCl}$. Die minimale Concentration von Natrium, die zur Erhaltung der Erregbarkeit von Muskeln eben ausreicht, bleibt also bei allen Natriumsalzen im Wesentlichen die gleiche. Es lässt sich also mit höchster Wahrseheinlichkeit sehliesseu, dass nur die Natrium-Ionen fur die Vorgänge der Erregungsleitung und der Muskelcontraction ron Bedeutung sind, währenddie Anionen und die nicht dissocirten Molekeln dabei nicht betheiligt sind oder höchstens eine ganz nebensächliche Rolle spielen. Verhielt es sich anders, so könnte die Natur der Anionen und der elektrisch neutralen Molekeln nicht ohne Einfluss auf diese Vorgänge sein.

Es wurde allerdings schon früher angedentet, dass, namentlich in etwas höheren Concentrationen, äquivalente Concentrationen verschiedener Natriumsalze bezüglich ihrer Wirkung auf die Muskeln keineswegs völlig mit einander übereinstimmen. Im Uebrigen hat schon $\mathrm{Nasse}^{1}$ ) vor mehr als 30 Jahren gefunden, dass die Muskeln in äquivalenten Lösungen verschiedener Natriumsalze ihre Erregbarkeit ungleich lang bewahren und zwar am längsten in Chlornatrium-Lösungen. Bei solchen Salzen wie ch lo r s a u rem Natri u m, benzoesaurem Natrium und Ferrocyannatrium ist dies leicht begreiflich, da das erste und letzte dieser Salze ziemlich reactionsfähig sind und die freie Benzoesäure, die immer in geringen Mengen abgespalten wird, äusserst leicht in die lebenden Muskelfasern eindringen wird. Schwer verständlich ist es dagegen, dass auch Natriunsulfat in Concentrationen, die mit $0,6 \% \mathrm{NaCl}$ isosmotisch sind, einen schädlichen Einfluss ausübt. Im Uebrigen leiden die motorischen Endplatten der Nerven viel früher und viel stärker unter dem Einfluss verschiedener dieser Salze (so z. B. des Natriumsulfats und des secundären Natriumphosphats) als die Muskelfasern selber.

Um einige dieser Verbältnisse anschaulicher zu machen, wird es vielleicht zweckmässig sein, die Protokolle einzelner der zahlreichen Versuche auszugsweise mitzutheilen.

1) Pflüger's Archiv Bd. 2 S. 114-121. 1869. 


\section{Versuch 12.}

Um 6.20 p. m. des 1. Juli 1901 Gastrocnemins + Nerv, die nach zweistündigem Liegen in $0,6 \% \mathrm{NaCl} 108 \mathrm{cg}$ wogen (noch in Gewichtszunahme begriffen) in $1,05 \% \mathrm{NaBr}$. (mit $0,6 \% \mathrm{NaCl}$ isosmotisch) übergeführt. Um 9.45 p. m. $115 \mathrm{cg}$; vom Nerven aus bei $18 \mathrm{~cm}$ Rollenabstand reizbar. Um 7.45 a. m. des 2. Juli $117 \mathrm{cg}$; rom Nerven aus bei $15 \mathrm{~cm}$ reizbar. Um $10.30 \mathrm{a} . \mathrm{m}$. ebenso. Um 9.50 p. m. des 2. Juli $120^{1 / 2} \mathrm{cg}$; sowohl direct wie indirect unerregbar. In diesem Versuche waren sowohl Muskel als auch der Nerr in der Lösung untergetaucht. Beide blieben ungcfäbr ebenso lange und ebenso leicht erregbar wie unter denselben Umständen in $0,6 \% \mathrm{NaCl}$.

\section{Versuch 13.}

Um 11.13 p. m. des 23. Januar 1902 Gastrocnemius + Nerv in $1,02 \mathrm{NaNO}_{3}$ (isosmotisch mit $0,7 \% \mathrm{NaCl}$ ) gesetzt. Sofort traten lebhafte Tetani und Zuckungen auf. Um 11.52 p. m. des 23. Januar Muskel immer noch in beständiger Unruhe. Um 9.55 a. m. des 24 . Januar vom Nerv aus völlig unreizbar (ursprünglich bei $27 \mathrm{~cm}$ Rollenabstand gut erregbar), direct bei $5 \mathrm{~cm}$ Rollenabstand reizbar, aber selbst bei $3 \mathrm{~cm}$ Contraction schwach.

\section{Versuch 14.}

Um 6.50 p. m. des 23. November 1901 Hinterfuss einer Rana esculenta nach $21 / 2$ stündigem Liegen in $0,7 \% \mathrm{NaCl}$ in $1,02 \% \mathrm{NaNO}_{8}$ (isosmotisch mit $0,7 \%$ NaCl) übergeführt. Kurze Zehenmuskeln im Anfang des Versuchs bei $10 \mathrm{~cm}$ gut contrahirbar. Um $10.40 \mathrm{p}$. m. des 23. November alle Fussmuskeln wie schon gleich nach ihrer Uebertragung in die $\mathrm{NaNO}_{3}$-Lösung in beständiger Unruhe. Kurze Zehenmuskeln bei $10 \mathrm{~cm}$ Rollenabstand leicht reizbar und alle Fussmuskeln contrahiren sich kräftig und in normaler Weise, wenn sie gereizt werden. Um 12.20 p. In. des 24 . November anch die kurzen Zehenmuskeln bei $10-12 \mathrm{~cm}$ Rollenabstand gut contrahirbar. Um $11.25 \mathrm{p}$. m. des 24 . November alle Fussmuskeln noch ziemlich gut erregbar, Contraction jedoch schwächer als unter normalen Umständen.

In diesen beiden letzten Versuchen sind die beständigen Zuckungen der Muskeln in den ersten Stunden vorwiegend einer erregenden und schliesslich schädigenden Wirknng des $\mathrm{NaNO}_{3}$ auf die motorischen Nervenendigungen und vielleicht auch auf die Nervenstämme zuzuschreiben. In $6 \%$ igen Rohrzuckerlösungen $+0,146 \% \mathrm{NaNO}_{3}$, oder in $5 \frac{1 / 2}{\%} \%$ igen Rohrzuckerlösungen $+0,2 \% \mathrm{NaNO}_{3}$ bleiben Muskeln ungefähr ebenso lange am Leben und ebenso erregbar wie in $0,7 \% \mathrm{NaCl}$.

\section{Versuch 15.}

Um 5.25 p. m. des 26. Juni 1901 Sartorius einer ơ Rana esculenta, der nach $1 \frac{1}{2}$ stündigem Liegen in $0,6 \% \mathrm{NaCl} 18 \mathrm{cg}$ wog, in $0,3 \% \mathrm{Na}_{2} \mathrm{SO}_{4}+1 \mathrm{Aq}$. (ungefähr isosmotisch mit $0,14 \% \mathrm{NaCl}$ ) $+6 \%$ Robyzuckerlösung (alles zusammen 
isosmotisch mit ca. $0,75-0,76 \%$ NaCl) übertragen. Um 8.05 p. m. des 26. Juni bei $12 \mathrm{~cm}$ Rollenabstand gut reizbar. Um $9.45 \mathrm{p}$. m. des 26. Juni $16 \%$ cg. Un $10.50 \mathrm{p}$. m. des 26 . Juni noch immer hei $12 \mathrm{~cm}$ Rollenabstand reizbar, Contraction bei $9-10 \mathrm{~cm}$ Rollenabstand recht energisch. - Um 8.20 a. m. des 27. Juni $16^{\frac{1}{2} \mathrm{cg}} \mathrm{c}$ n noch bei $5-6 \mathrm{~cm}$ Rollenabstand gut contrahirbar. Um 11.15 p. m. des 27 . Juni $17 \mathrm{cg}$, kaum noch erregbar. Um 9.40 p. m. des 29. Juni $17-17^{1 / 2} \mathrm{cg}$; unerregbar. Um 8.00 a. m. des 1 . Juli $15 \mathrm{cg}$.

\section{Tersuch 16.}

Un 5.12 p. m. des 23. Juni 1901 Gastrocnemius + Nerv, die nach kurzem Verweilen in $0,5 \% \mathrm{NaCl} 85 \mathrm{cg}$ wogen, in $1,5 \% \mathrm{Na}_{2} \mathrm{SO}_{4}+1 \mathrm{Aq}$. äbergeführt. Im Anfange des Versuchs Muskel bei $20 \mathrm{~cm}$ Rollenabstand rom Nerven aus reizbar. Um $9.40 \mathrm{p}$. m. des 23 . Juni $90 \mathrm{cg}$; vom Nerven aus nicht mehr reizbar, direct bei $6-7 \mathrm{~cm}$ Rollenabstand (statt 11-12 cni) reizbar, aber selbst bei völlig genäberten Rollen Contraction ziemlich scbwach. Um 8.20 a. m. des 24 . Juni $96 \mathrm{cg}$, vom Nerven aus unreizbar, direct erst bei $1-2 \mathrm{~cm}$ Rollenabstand deutliche Contraction. Um 10.52 p. m. des 24 . Juni $96 \mathrm{cg}$; unerregbar.

In $6 \%$ Rohrzucker $+0,3 \% \mathrm{Na}_{2} \mathrm{SO}_{4}+1 \mathrm{Aq}$ bleibt der Muskel auch vom Nerven aus wäbrend mehr als 10 Stunden reizbar, die Nerven oder vielmehr die Nervenendigungen verlieren indessen ihre Erregbarkeit auch in dieser Lösung früher als in $6 \%$ Rohrzucker $+0,15 \% \mathrm{NaCl}$. Die Nervenstämme werden durch $\mathrm{Na}_{2} \mathrm{SO}_{4}$ erst in viel höherer Concentration beschädigt, wie Versuche, in denen bloss der Nery in eine Lösung von $\mathrm{Na}_{2} \mathrm{SO}_{4}$ gesetzt wird, während der Muskel im dampfgesättigten Raume bleibt, zeigen.

\section{Yersuch 17.}

Um 10.45 p. m. des 27. Juni 1901 Sartorius einer $\delta$ Rana esculenta, der nach $4^{8 / 4}$ stündigem Verweilen in $0,6 \% \mathrm{NaCl} 22 \mathrm{cg}$ wog, in $0,25 \% \mathrm{Na}_{2} \mathrm{HPO}_{4}$ (isosmotisch mit ca. $0,135 \% \mathrm{NaCl}$ ) $+6 \%$ Rohrzucker übergeführt. Diese Lösung ist isosmotisch mit einer ca. 0,75\% NaCl-Lösung. Um $10.52 \mathrm{p}$. m. noch spontane Zuckungen, die sofort nach der Uebertragung einsetzten. Um 11.56 p. m. des 27. Juni $19^{3 / 4} \mathrm{cg}$; schon seit längerer Zeit keine spontanen Zuckungen mehr, bei $14 \mathrm{~cm}$ Rollenabstand reizbar, bei $11-12 \mathrm{~cm}$ Contraction sehr lebhaft. Um 10.00 a. m. des 28 . Juni $20 \mathrm{cg}$; bei $9 \mathrm{~cm}$ Rollenabstand Contraction ziemlich lebhaft. Um 3.15 p. m. $20^{1 / 2} \mathrm{cg}$; bei grösserer Rollenannäherung Contraction immer noch ziemlich lebhaft, Schwelle der Erregbarkeit aber viel höher geworden. Un 9.10 p. m. des 28 . Juni $21 \mathrm{cg}$; unerregbar, contrahirt und starr.

Noch etwas länger blieb ein Gastrocnemius in einer gleich zusammengesetzten Lösung erregbar. 


\section{Versuch 18.}

Um 3.02 p. m. des 6. October 1901 ein Gastrocnemiusnerv-Präparat, das nach $21 / 2$ stündigem Liegen in $0,6 \% \mathrm{NaCl}$ vom Nerven aus bei $33 \mathrm{~cm}$ Rollenabstand reizbar war, in $1.25 \% \mathrm{Na}_{2} \mathrm{HPO}_{4}$ (isosmotisch mit $0,675 \% \mathrm{NaCl}$ ) übergeführt. Sofort trat der Muskel in beständige, schwache Zuckungen und Tetani, obgleich das Präparat in der NaCl-Lösung völlig ruhig gewesen war. Um 3.42 p. m. Muskel immer noch in beständigen schwachen Znckungen begriffen. Um 5.30 p. m. des 6 . October noch bei $32 \mathrm{~cm}$ vom Nerven aus reizbar. Um 7.50 p. m. vom Nerven aus in seinem ganzen Verlaufe selbst bei völlig genäherten Rollen völlig unreizbar, direct bei $11 \mathrm{~cm}$ reizbar. Die, spontanen" Zuckungen hörten schon un ca. $6.00 \mathrm{p}$. m. auf, nachdem sie allmählich immer schwächer geworden waren. Um $12.0 \mathrm{i}$ p. m. des 7 . October direct bei $8 \mathrm{~cm}$ Rollenabstand eben merklich erregbar, aber auch bei $2 \mathrm{~cm}$ Contraction schwach. Um 7.40 p. m. bei völlig genäherten Rollen Contraction deutlich, aber sehr schwach. Um 3.45 p. m. des 8 . October unerreghar, aber noch sehr frisch aussehend und nicht eigentlich starr.

\section{Versuch 19.}

Um 6.07 p. m. des 3. October 1901 ein Gastrocnemiusnerv-Präparat so in einer Lösung von $1,25 \% \mathrm{Na}_{2} \mathrm{HPO}_{4}$ aufgehängt, dass nur der Nervenstamm in die Lösung untertauchte, der Muskel dagegen neben ca. $3 \mathrm{~mm}$ Nerv in dem nit Wasserdampf gesättigten Raume oberhalb der Lösung blieben. Der Muskel gerieth nach kurzer Zeit in schnell aufeinanderfolgende, schwächere Zuckungen, die lange Zeit anhielten, obgleich der Nerv bei der Präparation sehr sorgfältig behandelt wurde und der Muskel in $0,6 \% \mathrm{NaCl}$ vö!lig in Ruhe geblieben war. Um 7.40 p. m. vom proximalen (centralen) Ende des Nerven aus bei $40-45 \mathrm{~cm}$ Rollenabstand völlig sicher in Erregung zu versetzen. Um 10.50 p. m. ca. $1^{1 / 2} \mathrm{~cm}$ des Nerven am proximalen Ende unerregbar, weiter distalwärts schon bei $20-25 \mathrm{~cm}$ Rollenabstand reizhar. Tm 8.25 a. m. des 4. October Muskel von der ganzen distalen Hälfte des Nerven aus noch bei einem Rollenabstand von $20-25 \mathrm{~cm}$ reizbar, in nächster Nähe des Muskels löst schon eine Reizung des Nerven bei $35 \mathrm{~cm}$ Rollenabstand eine Contraction aus. Um $3.02 \mathrm{p}$. m. des 4. October vom distalen Ende des Nerven immer noch sicher reizbar bei $35 \mathrm{~cm}$ Rollenabstand, von der ganzen distalen Hälfte bei $20-25 \mathrm{~cm}$. Um $9.30 \mathrm{p.} \mathrm{m}$. des 4. October lässt sich eine Contraction nur vom distalen Ende des Nerven in einer Länge von ca. $12 \mathrm{~mm}$ auslösen, an einer Stelle unmittelbar vor dem Eintreten des Nerven in den Muskel aber noch bei $25 \mathrm{~cm}$ Rollenabstand; Muskel direct bei 10-11 cm Rollenabstand contrahirbar. Um 8.40 a. m. des 5. October Muskel sowohl indirect wie direct unerregbar.

Aus dem letzten Versuche (19) ergibt sich, dass der Nervenstamm in einer Lösung von $1,25 \% \mathrm{Na}_{2} \mathrm{HPO}_{4}$ eher länger erregbar bleibt als in $0,7 \% \mathrm{NaCl}$, während die Enden der motorischen Nerven nach wenigen Stunden (Versuch 18) oder bei kleineren Muskeln selbst innerhalb einer Stunde gelähmt oder getödtet werden, wenn 
der Muskel in der Lösung verweilt. Die Muskelfasern selbst werden durch diese Lösung nur sehr wenig beschädigt, und in geringeren Concentrationen von $\mathrm{Na}_{2} \mathrm{HPO}_{4}$ (Gemischen von Robrzucker und $\mathrm{Na}_{2} \mathrm{HPO}_{4}$ ) gar nicht. Die reizende Wirkung des $\mathrm{Na}_{2} \mathrm{HPO}_{4}$ auf die Nervenstämme und im Anfange des Versuchs auf die Nervenendigungen ist fast sicher bedingt durch die schwach alkalische Reaction des secundären Natriumphosphats (also durch die Gegenwart von $\mathrm{OH}-$ Ionen), da die Lösungen aller schwach alkalisch reagirenden Salze ähnlich wirken, sofern die betreffenden Salze nicht einen specifisch lähmenden Einfluss ausüben.

\section{Versuch 20 .}

Um 9.00 p. m. des 27. Juni 1901 Sartorius einer $\delta$ Rana esculenta, der nach $34 / 2$ stündigem Verweilen in $0,6 \% \mathrm{NaCl} 22 \mathrm{cg}$ wog, in $0,144 \% \mathrm{Na} \mathrm{HCO}_{3}$ isosmotisch mit $0,1 \% \mathrm{NaCl}$ ) $+6 \%$ Rohrzucker gesetzt. Um $9.58 \mathrm{p}$. m. des 27. Juni $20-20^{1 / 4} \mathrm{cg}$. Um 11.10 p. m. noch schwache „spontane“ Zuckungen. Um 11.50 p. m. starke, spontane Zuckungen in rascher Folge. Um $9.57 \mathrm{a.} \mathrm{m}$. des 28. Juni $201_{14}-20 \% / 2 \mathrm{cg} ;$ noch bei $12 \mathrm{~cm}$ Rollenabstand Contraction lebhaft. Um $2.47 \mathrm{p}$. m. des 28 . Juni $201 / \mathrm{gg}$; bei $12 \mathrm{~cm}$ noch gut contrahirbar. Um $8.55 \mathrm{p} . \mathrm{m}$. des 28. Juni $23 \mathrm{cg}$; nur noch spturweise erregbar. Um $9.25 \mathrm{p} . \mathrm{m}$. des 29. Juni unerregbar, $25 \mathrm{cg}$.

\section{Yersuch थ1.}

Um 9.07 p. m. des 27. Juni 1901 Gastrocneminsnerv-Praparat, das rom Terven aus bei ca. $20 \mathrm{~cm}$ Rollenabstand reizbar war, in $6 \%$ Rohrzucker $+0,144 \% \mathrm{NaHCO}_{3}$ übertragen. Um 2.50 p. m. des 28 . Juni vom Nerven ans erst bei $3 \mathrm{~cm}$ Rollenabstand reizbar und auch dann nur vom distalen Ende des Nerven, direct bei $14 \mathrm{~cm}$ contrahirbar. Um $9.00 \mathrm{p}$. m. vom Nerven aus unreizbar, directe Erregbarkeit noch erhalten, aber stark abgenommen. Um 9.22 p. m. des 29. Juni starr.

Aus diesen Versuchen ist zu sehen, dass $\mathrm{NaHCO}_{3}$ in Concentrationen von $0,15 \%$ kaum eine schädliche Wirkung auf die Muskelfasern ausübt und selbst die Nervenendigungen nur sehr langsam schädigt. In etwas höheren Concentrationen werden die Nerrenendigungen rasch getödtet, die Nervenstämme nicht oder sehr langsam, aber auca sie werden in fast beständiger Erregung gehalten.

\section{Versuch ․‥}

Um 3.04 p. m. des 2. Mai 1902 ein Hinterfuss ron Rana esculenta, dessen knuze Zehenmuskeln bei $8-10 \mathrm{~cm}$ und dessen übrige Muskeln bei $16-17 \mathrm{~cm}$

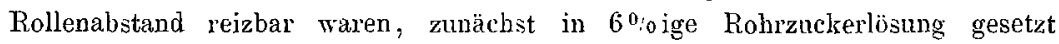
und dann, nacbdem die kurzen Zehenmuskeln schon seit mehr als $1^{1 / 9}$ Stunden rollig unerregbar gewesen waren, um $5,04 \mathrm{p} . \mathrm{m}$. in $0,2 \% \mathrm{NaClO}_{3}$ (isosmotisch 
mit $0,11 \% \mathrm{NaCl})+6 \%$ Rohrzucker gesetzt. Um 7.50 p. m. des 2. Mai kurze Zehenmuskeln wieder bei $8-10 \mathrm{~cm}$ reizbar, die übrigen Fussmuskeln, die nie unerregbar geworden sind (wegen des zu kurzen Aufenthalts in der reinen Zuckerlösung) bei $16-17 \mathrm{~cm}$ Rollenabstand gut contrahirbar. Um $10.52 \mathrm{p} . \mathrm{m}$. des 2. Nai kurze Zehenmuskeln bei $8-10 \mathrm{~cm}$, die übrigen Fussmuskeln bei 16-17 cin gut contrahirbar. Um 10.20 a. $\mathrm{m}$. des 3. Mai kurze Zehenmuskeln erst bei $2-3 \mathrm{~cm}$ reizbar und Contraction schwach, die übrigen Fussmuskeln noch bei 14-15 cm Rollenabstand reizbar und Contraction ziemlich stark, Lösung schwach, aber deutlich sauer reagirend, was gestern nicht der Fall war. Um 11.05 p. m. des 3. Mai alle Muskeln unerregbar.

\section{Tersnch 23.}

Um 7.15 p. m. des 23. Januar 1902 ein Hinterfuss von Rana esculenta. dessen Muskeln durch Verweilen in $6 \%$ Rohrzucker völlig unerregbar geworden waren, in $1,62 \%$ Natriumacetat $\mathrm{NaC}_{2} \mathrm{H}_{3} \mathrm{O}_{2}+3 \mathrm{Aq}$ (isosmotisch mit ca. $0,7 \% \mathrm{NaCl}$ ) übergefübrt. Schon nach 5 Minuten die kurzen Zehenmuskeln wieder gut erregbar. - Jm 10.30 p. m. des 23. Januar alle Muskeln ebenso gnt contrahirbar wie in $0,7 \% \mathrm{NaCl}$, die kurzen Zehenmuskeln z. B. bei $10 \mathrm{~cm}$ Rollenabstand. Um 12.40 p. m. des 24. Januar alle Muskeln ebenso gut erregbar wie im Anfang des Versuchs. - Um 11.27 des 24. Januar kurze Nuskeln der mittleren Zehe bei $8 \mathrm{~cm}$ Rolienabstand gut contrahirbar, die anderen kurzen Zehenmuskeln nur noch wenig erregbar, die übrigen Fussmuskeln contrahiren sich ziemlich lebhaft bei $10-12 \mathrm{~cm}$ Rollenabstand. Die Lösung reagirte sehr schwach alkalisch (wegen geringer hydrolytischer Spaltung des Natriumacetats).

\section{Yersuch 24 .}

Um 11.27 p. m. des 23 . Januar 1902. Gastrocnemius + Nerv einer Rana esculenta nach $7{ }^{1}$ stündigem Verweilen in $0,7 \% \mathrm{NaCl}$ in $1,62 \%$ Natriumacetat $\left(\mathrm{NaC}_{2} \mathrm{H}_{3} \mathrm{O}_{2}+3 \mathrm{Aq}\right)$ übergeführt. Unmittelbar vor der Uebertragung war der Muskel vom Nerv aus bei $30 \mathrm{~cm}$ Rollenabstand erregbar. Nach einiger Zeit ziemlich häufige „spontane“ Zuckungen. - Um 10.05 a. m. des 24. Januar vom Nerv aus bei $25 \mathrm{~cm}$ Rollenabstand contrahirbar, direct bei $12 \mathrm{~cm}$. Auch „spontane Zuckungen ziemlich häufig (Folge der schwachen Alkalescenz der Lösung). Um 11.20 p. m. des 24. Januar vom Nerv aus völlig unreizbar, direct erst bei $3-4 \mathrm{~cm}$ Rollenabstand.

\section{Versuch æ25.}

Um 5.00 p. m. des 2. Mai 1902 ein Hinterfuss vom Rana esculenta nach 7 stïndigem Verweilen in $0,7 \% \mathrm{NaCl}$ in $0.25 \%$ benzoësaures Natrium (isosmotisch mit $0,1 \% \mathrm{NaCl})+60_{0}^{\circ}$ Rohrzucker übergefuhrt. - Um 8.50 p. m. kurze Zehenmuskeln merklich erregbar, aber Contraction sehr schwach; die anderen Fussmuskeln bei 8-9 cm Rollenabstand reizbar. Durch Zusatz einer Spur von $\mathrm{MgO}$ wurde die Erregbarkeit etwas aufgebessert. -- Um 11.03 p. m. des 2. Mai ebenso. -- Um 11.10 a. m. des 3. Mai Muskeln immer noch etwas erregbar.

In diesem Versuche hat die Benzoësäure eine mehr oder weniger lähmende Wirkung ausgeïbt. 


\section{Fersuch 26 .}

Um 10.37 p. m. des 27. Mai 1902 ein Hinterfuss von Rana esculenta, dessen Muskeln durch längeres Liegen in Rohrzucker röllig unerregbar gewrorden waren, in $5 \%$ Rohrzucker $+0,4 \%$ Ferricyannatrium $\left(\mathrm{Na}_{3} \mathrm{Fe}(\mathrm{CN})_{6}+2 \mathrm{Aq}\right)$ übergeführt. Um 12.35 a. m. des 28. Mai kurze Zehenmuskeln bei $10 \mathrm{~cm}$ Rollenabstand gut contrahirbar. - Um 3.15 p. m. des 28. Mai kurze Zehenmuskeln bei 9-10, die übrigen Fussmuskeln bei $15-16 \mathrm{~cm}$ Rollenabstand gut contrahirbar. - Um 10.52 p. m. des 28. Mai die kurzen Zehenmuskeln bei 6 , die übrigen Fussmuskeln bei $14-15 \mathrm{~cm}$ reizbar; Contraction etwas schwächer als normal.

Wie aus dem letzten Versuche zu ersehen ist, wirkt Ferricyannatrium (rothes Blutlaugensalz, bei dem das Kalium durch Natrium ersetzt ist) in der angewandten Concentration kaum schädlich auf die Muskeln. Ferrocyannatrium (dem gelben Blutlaugensalz entsprechend) stellt zunächst die Erregbarkeit der Muskeln ebenfalls wieder her, entfaltet aber viel früher eine schädigende Wirkung auf die Muskeln als das Ferricyannatrium.

\section{Können andere Kationen das Natriumion in seiner Eigenschaft, die Erregbarkeit der Muskeln zn erhalten, vertreten?}

Bei der experimentellen Untersuchung dieser Frage können natürlich nur die Salze der Alkalien, der Erdalkalien und des Ammoniaks in Betracht kommen, da die Salze aller Schwermetalle zu den allgemeinen Protoplasmagiften gehören. Es war nun zunächst nöthig, eine grosse Reihe von Vorversuchen über das Verhalten der Muskeln gegenüber den verschiedenen Salzen der Leichtmetalle anzustellen, welche zu vielen neuen Ergebnissen führte, die etwas abseits von dem eigentlichen Thema dieser Arbeit liegen und daher in einer besonderen Mittheilung besprochen werden sollen. An dieser Stelle genügen folgende Angaben: Alle Salze des Kaliums, des Rubidiums und des Caesiums lähmen schon in Concentration, die einer $0,07 \%$ igen Natriumchloridlösung äquivalent sind, die Muskeln vollständig, und auch in niedrigeren Concentrationen können sie die Natriumsalze nicht einmal theilweise ersetzen. In $6 \%$ Rohrzucker $+0,08 \%$ $\mathrm{NaCl}+0,02 \% \mathrm{KCl}$ sind Muskeln allerdings etwas erregbarer als in $6 \%$ Rohrzucker $+0,08 \% \mathrm{NaCl}$ ohne Zusatz des Kaliumchlorids, wie Spuren von Kalium und Calciumsalzen überhaupt eine günstige Wirkung auf die Muskeln ausüben; in $6 \%$ Rohrzucker $+0,06 \%$ $\mathrm{NaCl}+0,02 \% \mathrm{KCl}$ bleiben aber Muskeln völlig unerregbar, und ein grösserer Zusatz von $\mathrm{KCl}$ wirkt schädlich. 
Calcium- und Strontiumehlorid, die zu einer Rohrzuckerlösung gesetzt werden, verzögern den Eintritt des unerregbaren Zustandes der Muskeln, was indessen wenigstens zum Theil darauf zurückzuführen ist, dass sie die Exosmose des Natriumchlorids aus der Zwischenflüssigkeit der Muskeln verlangsamen. Letzteres rührt daher, dass durch den Zusatz eines Chlorids zu der Zuckerlösung das Diffusionspotential (Diffusionsgefälle) der Chlorionen zwischen der Lösung, welche die einzelnen Muskelfasern umspülen, und der Lösung, die den ganzen Muskel umgibt, erniedrigt wird. Diese Erniedrigung des Diffusionspotentials der Chlorionen bedingt zunächst eine langsamere Exosmose der Chlorionen aus den Muskeln. Da nun die Geschwindigkeit der Chlorionen grösser ist als diejenige der Natriumionen, eine unabhängige Wanderung von Kationen und Anionen aber nicht möglich ist, resp. nur in minimalem Grade stattfinden kann, wegen des bei einer ungleichmässigen Vertheilung der Kationen und Anionen sofort auftretenden elektrischen Potentials, so schleppen die lebhafteren Chlorionen die trägeren Natriumionen mit. Hierdurch wird die Eigengeschwindigkeit der Chlorionen herabgesetzt, die Wanderung der Natriumionen aber dafür beschlemigt, ähnlich wie die Geschwindigkeit eines mit Segeln versehenen Lastschiffes durch einen Schleppdampfer erhöht werden kann, während die ursprüngliche Geschwindigkeit des Schleppdampfers durch die Spannung des Schlepptaues herabgesetzt wird. Wenn also die Exosmose der Chlorionen atis den Muskeln verlangsamt wird, verzögert sich zugleich die Exosmose der Natriumionen (wenn man den soeben angezogenen Vergleich ausspinnen wollte, könnte man hinzufügen, ähnlich wie die mittlere Geschwindigkeit einer gegebenen Anzahl Segel-Lastschiffe herabgesetzt wird, wenn die Anzahl der zur Verfügung stehenden Schleppdampfer eine kleinere ist).

Wenn Muskeln in reinen Rohrzuckerlösungen bereits völlig unerregbar geworden sind, kann die Erregbarkeit durch einen Zusatz von Calcium-, Strontium - oder Magnesiumsalzen nicht wieder hergestellt werden, obgleich diese Salze nur wenig giftig sind und Kochsalzlösungen in bedeutend grösseren Mengen beigemischt werden können, als einer $0,07 \%$ igen Chlornatriumlösung äquivalent wären, ohne die Contractionsstärke der Muskeln wesentlich zu beeinträchtigen. - Bei der grossen Giftigkeit der löslichen Bariumsalze für Muskeln und Nerven war ohne Weiteres vorauszusehen, dass diese Salze die Natriumsalze nicht vertreten könnten. 
Ebensowenig wie Kalium-, Rubidium-, Caesium-, Magnesium-, Calcium- und Strontiumsalze für sich allein, d. h. nur im Verein mit der Lösung eines Nichtleiters (wie Rohrzucker, Traubenzucker, Alanin) die Erregbarkeit eines Muskels erhalten resp. wieder herstellen können, vermögen beliebige Gemische dieser Salze die Erregbarkeit des Muskels zu erhalten.

Ganz anders liegen dagegen die Verhältnisse bei den Lithium salzen, indem diese in der That die Natriumsalze ersetzen können, wenn sie auch freilich in etwas böheren Concentrationen auf die Muskeln und noch mehr auf die Nerven allmählich schädlich wirlken.

Im Folgenden sollen einige Versuche mit Lithiumsalzen mitgetheilt werden.

\section{Versuch 2:.}

Um 3.10 p. m. des 12. Juli 1901 wurde ein Sartorius, der nach ${ }^{3 / 1}$ stündigem Verweilen in $0,6 \% \mathrm{NaCl} 18 \mathrm{cg}$ wog und bei $98 \mathrm{~cm}$ Rollenabstand reizbar war, in $7 \%$ Rohrzucker übergeführt. In den ersten 4 Minuten häutige "spontane" Zuckongen, die bald seltener wurden und dann ganz aufhörten. - Von $3.40 \mathrm{p.m}$. an selbst bei völlig genäherten Rollen nicht mehr reizbar. - Un 4.35. p. m. $161 / 2 \mathrm{cg}$; darauf sofort in $0.435 \%$ Lithiumchlorid (isosmotisch mit $0,6 \% \mathrm{NaCl}$ ) übergeführt. Schon um $4.44 \mathrm{p}$. m (also nach etwa 8 Minuten) bei $10 \mathrm{~cm}$ Rollenabstand reizbar und bei $7 \mathrm{~cm}$ Contraction recht stark. - Um 4.55 p. m. $17^{3 / 4} \mathrm{cg}$; Erregbarkeit noch zugenommen. - Um 7.27 p. m. vollständig unerregbar und ziemlich sicher todt, $201 / 2 \mathrm{cg}$. - Um $7.35 \mathrm{p}$. m. in $0,6 \% \mathrm{XaCl}$ zurückgesetzt. Um $9.25 \mathrm{p} . \mathrm{m}$. völlig unerregbar, $23 \mathrm{cg}$. $-\mathrm{Um} 8.43$ a. m. des 13 . Juli $26 \% \frac{1}{2} \mathrm{cg}$, todtenstarr.

\section{Versuch 29.}

Um 5.27 p. m. des 5. Juli 1901 wurde ein Sartorius, der nach 2 stïndigem Liegen in $6 \%$ Robrzucker $201 / 2 \mathrm{cg}$ wog und völlig unerregbar geworden war, in $0,22 \% \mathrm{LiCl}$ (isosmotisch mit $0,3 \% \mathrm{NaCl}$ ) + 3\% Pohrzucker ühergeführt. Um 6.01 p. m. $20^{1 / 2}-20^{3 / 4}$ cg. - Um 6.03 p. m. schon bei $10 \mathrm{~cm}$ Rollenabstaud reizbar, bei $5 \mathrm{~cm}$ Contraction recht lebhaft. - Um 7.17 p. m. 201/2 cg. - Um 8.55 p. m. $20^{1 / 2} \mathrm{cg}$, noch bei $12 \mathrm{~cm}$ Pollenabstand reizbar, bei $5-6 \mathrm{~cm}$ Contraction sehr lebhaft. - Um 11.30 p. m. $20 \% / \mathrm{cg}$, bei $10 \mathrm{~cm}$ Rollenabstand reizbar, bei $8 \mathrm{~cm}$ Contraction lebhaft. - Um 7.40 a. m. des 6 . Juli $20 \mathrm{cg}$, noch bei 6 cm Rollenabstand reizbar. - Um 2.00 p. m. $20^{1 / 2}-21 \mathrm{cg}$, noch etwas erregbar. - Um 8.33 a. m. des 7. Juli $22 \mathrm{eg}$; starr.

\section{Versuch 29 .}

Um 7.00 p. m. des 5. Juli 1901 Gastrocnemius (sammt Nerven) einer Rana esculenta, der nach mehr als 4 stiundigem Verweilen in 6\% Rohrzucker $167 \mathrm{cg}$ wog (an Gewicht noch etwas zunehmend), in $3 \%$ Pohrzucker $+0,22 \%$ Li i l gesetzt $(0,22 \% \mathrm{LiCl}$ isosmotisch mit $0,3 \% \% \mathrm{NaCl})$. Zur Zeit der Ueberführung war der Muskel sowohl direct wie vom Nerven aus völlig unerregbar. - Um 
9.25 p. m. des 5. Juli $170 \mathrm{cg}$; direct schon bei $12 \mathrm{~cm}$ Rollenabstand reizbar, vom Nerven aus nur sehr wenig reizbar. - Um 11.35 p. m. des 5 . Juli $170 \mathrm{cg}$; direct bei 12-13 cm Rolienabstand reizbar, vom Nerven aus unreizbar. - Um 8.00 a. m. des 6 . Juli $173 \mathrm{cg}$; direct bei $4^{1 / 2} \mathrm{~cm}$ reizbar, aber Contraction ziemlich schwach. - Um $2.00 \mathrm{p}$. m. des 6 . Juli unerregbar und zweifellos todt. Um 8.20 a. m. des 7 . Juli $184 \mathrm{cg}$, starr.

\section{Yersuch 30.}

Um 2.30 p. m. des 12. Juli 1901 Gastrocnemius (sammt Nerv) einer Rana esculenta, der nach 15 Minuten Verweilen in $0,6 \% \mathrm{NaCl} 85 \mathrm{cg}$ wog und vom Nerven aus bei $24 \mathrm{~cm}$ Rollenabstand reizbar war, in $7 \%$ Rohrzucker übergeführt. Um 4.40 p. m. $86 \mathrm{cg}$; völlig unreizbar. Um 4.43 p. m. in $0,435 \% \mathrm{LiCl}$ (isosmotisch mit $0,6 \% \mathrm{NaCl}$ ) übergeführt. Um 5.20 p. m. $90 \frac{1 / 2}{\mathrm{cg}}$; direct bei $8 \mathrm{~cm}$ Rollenabstand reizbar, bei $4 \mathrm{~cm}$ Contraction ziemlich stark; vom Nerven aus erst bei $4 \mathrm{~cm}$ Rollenabstand reizbar und selust bei völlig genäherten Rollen Contraction sehr schwach. Um 7.45 p. m. des 12. Juli $981 / 2 \mathrm{cg}$; vom Nerven aus völlig unreizbar, direct bei $7^{1 / 2} \mathrm{~cm}$ reizbar, aber auch bei grösster Rollenannäherung Contraction nur mässig stark. Um $9.30 \mathrm{p}$. m. nicht oder kaum mehr erregbar, $100^{1 / 2} \mathrm{cg}$. Darauf in $0,6 \% \mathrm{NaCl}$ übergeführt. Um 8.40 a. m. des 13. Juli $102 \mathrm{cg}$; unerregbar. Versuch abgebrochen.

\section{Versuch 31.}

Um 2.50 p. nt. des 27. Juli 1901 ein Gastrocnemiusnerv-Präparat, das im Anfange des Versuchs vom Nerven aus bei $24 \mathrm{~cm}$ Rollenabstand reizbar war, so in $0,218 \% \mathrm{LiCl}$ (isosmotisch mit $0,3 \% \mathrm{NaCl}$ ) $+30 \%$ Rohrzucker gesetzt, dass nur der Nerv in die Lösung untertauchte, während der Muskel selber sich im dampfgesättigten Raume oberhalb der Lösung befand. Um 9.35 p. m. (also nach ca. $61 / 2$ Stunden) nur von der distalen (peripherischen) Hälfte des Nerven aus reizbar und auch von hier aus erst bei $2-3 \mathrm{~cm}$ Rollenabstand reizbar, direct bei $12 \mathrm{~cm}$ reizbar. Um 9.38 p. $\mathrm{m}$. das ganze Präparat in die Lösung grebracht. Um 11.30 p. m. des $2 \bar{T}$. Juli von der distalen Hälfte des Nerven aus bei $2 \mathrm{~cm}$, direct bei $10 \mathrm{~cm}$ reizbar, bei grösserer liollenannäherung Contraction bei der directen Reizung recht lebhaft. Um 7.45 a. m. des 28. Juli vom Nerven aus völlig unreizbar, direct bei $10 \mathrm{~cm}$ reizbar, Contraction bei grösserer Rollenannäherung noch lebhaft. $\operatorname{Um} 4.00 \mathrm{p}$. m. des 28 . Juli Muskel nur noch sehr wenig erregbar.

\section{Versuch Bg.}

Um 2.30 p. m. des 25. Juli 1901 ein Gastrocnemiusnerv-Präparat so in $0,218 \% \mathrm{LiCl}+0,3 \% \mathrm{NaCl}$ gesetzt, dass nur der Nerv bis zu $2 \mathrm{~mm}$ vor seiner Fintrittsstelle in den Muskel in die Lösung untertauchte, der Muskel selber dagegen in mit Wrasserdampf gesättigter Luft hing. Um 5.00 p. m. noch bei $28 \mathrm{~cm}$ Rollenabstand reizbar wie im Anfange das Versuchs. Um $11.35 \mathrm{p.m}$. vom Nerven aus erst bei $8 \mathrm{~cm}$ Rollenabstand reizbar. Um 9.00 a. m. des 26. Juli vom Nerven aus völlig unreizbar.

E. Pflüg er, Archiv für Physiologie. Bd. 92. 


\section{Tersuch :38.}

Um 2.17 p. m. des 2. August 1901 Gastrocnemiuspräparat, das nach 2/2stündigem Liegen in $0,6 \% \mathrm{NaCl}$ vom Nerren aus bei $16 \mathrm{~cm}$ reizbar war, in $0,145^{\circ} \%$ LiCl (isosmotisch mit $0,2 \% \mathrm{NaCl}$ ) $+0,4 \% \mathrm{NaCl}$ übergeführt. Nerv im Anfang des Versuchs bei $16-18 \mathrm{~cm}$ gut reizbar. - Um $6.00 \mathrm{p} . \mathrm{m}$. vom Xerven aus, selbst ganz am proximalen (centralen) Ende, bei $10-12 \mathrm{~cm}$ Rollenabstand reizbar. - Um 8.10 p. m. des 2. August nur noch rom distalen Ende des Nerven aus reizbar und auch hier nur bei fast völlig genäherten Rollen.

Aus diesen und anderen Versuchen folgt, dass die Muskeln durch $0,435 \% \mathrm{LiCl}$ allerdings ziemlich schnell geschädigt werden; durch $0,218^{\circ} \% \mathrm{LiCl}$ ist die Schädigung der Muskeln eine recht langsame und durch $0,145 \%$ oder weniger ist eine schädigende Wirkung des Lithiums auf die Muskeln kaum mehr wahrzunehmen. Auf die motorischen Nervenenden und in geringerem Grade auf die Nervenstämme wirkt $0,218 \% \mathrm{LiCl}$ in renigen Stunden lähmend oder tödtlich, $0,145 \%$ entschieden schädlich.

Von Wichtigkeit schien die Feststellung der minimalen Concentration des LiCl, die noch zur Erhaltung der Erregbarkeit der Muskeln ausreicht. Diese Concentration ergab sich zu fast genau $0.05^{\circ} \%$, wie die beiden folgenden Versuche zeigen.

\section{Versuch 34.}

Um 9.45 p. m. des 13. Mai wurde der Hinterfuss einer Rana esculenta nach mehrstiundigem Liegen in $0,7 \% \mathrm{NaCl}$ in $6 \%$ Rohrzucker $+0,06 \%$ LiCl gesetzt. Kurze Zehenmuskeln in Anfang des Versuchs bei $9-10 \mathrm{~cm}$ Rollenabstand ausgezeichnet contrahirbal. - Um $11.53 \mathrm{p}$. m. alle kurzen Zehennuskeln noch contrahirbar, aber Contraction etwas träge und ziemlich schwach. - Um 8.30 a. m. des 14. Mai Contraction der kurzen Zehenmuskeln bei $2-3 \mathrm{~cm}$ Rollenabstand zwar träge, aber nicht schwach, und schon bei $7 \mathrm{~cm}$ eben dentlich contrahirbar; die übrigen Fussmuskeln bei $8 \mathrm{~cm}$ Rollenabstand merklich contrahirbar und bei $6 \mathrm{~cm}$ Contraction ziemlich stark. -- Um $7.50 \mathrm{p.} \mathrm{m}$. ebenso. Versuch abgebrochen.

\section{Tersuch 35.}

Um 9.43 p. m. des 13 . Jai 1902 ein Hinterfuss ron Rana esculenta in $6 \%$ Rohrzucker $+\mathbf{0 , 0 5} \% \mathrm{LiCl}$ übergeführt. Im Anfaug des Tersuchs die kurzen Zehenmuskeln bei $9-10 \mathrm{~cm}$ ausgezeichnet contrahirbar. - Um $11.50 \mathrm{p} . \mathrm{m}$. kurze Zehenmuskeln bei röllig genäherten Rollen eben merklich contrahirbar; die übrigen Fussmuskeln noch bei $16-17 \mathrm{~cm}$ Rollenabstand contrahirbar ( $\mathrm{NaCl}$ noch nicht völlig exosmirt aus diesen letzteren Muskeln). - Um 11.10 a. m. des 14. Mai alle Muskeln bei völlig genäherten Rollen eben merklich erregbar. Versuch abgebrochen. 
Aus diesen zwei Versuchen ergibt sich, dass die minimale Concentràtion von $\mathrm{LiCl}$, die zur Erhaltung der Erregbarkeit ausreicht, etwas niedriger als bei $\mathrm{NaCl}$ liegt, wenn man nach Gewichtsconcentrationen rechnet, dagegen mit der minimalen Concentration von $\mathrm{NaCl}$ genau übereinstimmt, wenn man nach Molekülconcentrationen zäblt, was hier zweifellos rationeller erscheint.

\section{Versuch 36.}

Um 4.02 p. m. des 30. Mai 1902 wurde ein Hinterfuss von Rana esculenta, dessen kurze Zehenmuskeln durch Verweilen in $7 \%$ Rohrzucker völlig unerregbar geworden waren, in $5 \%$ Rohrzucker $+0,16 \%$ Lithiumsulfat übergeführt. Um 11.05 p. m. kurze Zehenmuskeln bei $8-9 \mathrm{~cm}$ Rollenabstand contrahirbar, die anderen Fussmuskeln bei $15-16 \mathrm{~cm}$; Erregbarkeit aller Muskeln cntschieden etwas geringer als in einer äquivalenten Lösung von $\mathrm{NaCl}+$ Rohrzucker. Um 8.45 a. m. des 31. Mai kurze Zehenmuskeln bei $6 \mathrm{~cm}$ Rollenabstand reizbar, aber Contraction ziemlich schwach; die übrigen Fussmuskeln bei $10-12 \mathrm{~cm}$ reizbar und noch bei $8 \mathrm{~cm}$ nur mässig stark contrahirbar. - Um $10.40 \mathrm{a}$. m. des 1. Juni alle Muskeln völlig unerregbar.

\section{Versuch 37.}

Um 5.55 p. m. des 24. Juli 1901 wurde ein Gastrocneminsnerv-Präparat so in eine Lösung von $3 \%$ Rohrzucker $+0,241 \% \mathrm{Li}_{2} \mathrm{SO}_{4}$ gesetzt, dass nur der Nerv in die Lösung tauchte. - Um 8.17 p. m. vom Nerven aus noch bei einem Rolienabstand von mebr als $38 \mathrm{~cm}$ reizbar. - Um 10.12 p. m. ebenso; der Muskel führt übrigens zahlreiche schwache, "spontane" Zuckungen aus, was zweifellos auf einer geringen reizenden Wirkung der Lösung auf den Nervenstamm beruht. - Um 9.35 a. m. des 25 . Juli vom proximalen Nervenende aus unreizbar, vorn distalen Ende (bis zu $21 / 2 \mathrm{~cm}$ vom Muskel entfernt) bei $6 \mathrm{~cm}$ Rollenabstand reizbar. Darauf auch der Muskel in die Lösung gesetzt. - Um 11.40 p. m. des 25. Juli noch immer bei $6 \mathrm{~cm}$ Rollenabstand reizbar. - Um $9.10 \mathrm{a}$. m. des 26. Juli merregbar.

Wie Lithiumchlorid und Lithiumsulfat können andere Salze des Lithiums, z. B. Lithiumnitrat, secundäres Li thiumphosphat, Lithiumacetat dazu dienen, um die Erregbarkeit von Muskeln in Lösungen von nicht eindringenden Non-Elektrolyten zu erhalten resp. die in diesen Lösungen verloren gegangene Erregkeit wieder herzustellen. Bei allen diesen Salzen bleibt die minimale Concentration der Lithiumionen, welche die Erregbarkeit gerade noch erbält, fast oder ganz die gleiche, und es ist evident, dass die Contraction resp. die Erregungsleitung der Muskeln allein von der Anwesenheit der Lithiumionen, nicht von derjenigen der Anionen abhängt. Die etwas ungleichen Wirkungen äquivalenter Concen- 
trationen der verschiedenen Lithiumsalze, namentlich in höheren Concentrationen muss wie bei den entsprechenden Natriumsalzen auf einer secundären Wirkung der Anionen (vielleicht auch der nichtdissociirten Molekeln) der betreffenden Salze beruhen.

Unterminimale Concentrationen von Natrium- und Lithiumsalzen können sich zu überminimalen Wirkungen addiren, so genügt $z$. B. die Lösung von $6 \%$ Rohrzucker $+0,04 \% \mathrm{NaCl}+0,03 \% \mathrm{LiCl}$, um die Erregbarkeit der Muskeln zu erhalten, nicht aber die Lösung $6 \%$ Rohrzucker $+0,04 \% \mathrm{NaCl}$ oder die Lösung $15 \%$ Rohrzucker $+0,03 \% \mathrm{LiCl}$ einzeln für sich

\section{Schlussbetrachtungen.}

Im Vorausgehenden habe ich mich, etwa von dem einleitenden Paragraphen abgesehen, auf eine einfache Wiedergabe von Versuchen und die Darlegung der unmittelbar aus derselben zu ziehenden Schlüsse beschrünkt, ohne jede weitergehende theoretisehe Deutung derselben zu versuchen. Auch in den folgenden Betrachtungen werde ich mir in dieser Hinsicht grosse Einschränkungen auferlegen und mich mit einigen Andeutungen und Fragestellungen beguügen.

Zunächst möge hervorgehoben werden, dass durch diese Untersuchung wohl zum ersten Mal eine ganz specifische Function des Natriums im Wirbelthierorganisnus aufgedeckt worden ist. Bisher, wohl in wesentlicher Uebereinstimmung mit der Mehrzahl der Physiologen, hatte ich immer angenommen, dass das Natriumchlorid nur dazu diene, um ren osmotischen Druck der Säfte auf einer gewissen Höhe zu erhalten und dass speciell diese Verbindung zu dem oben genannten Zwecke verwendet worden ist, weil sie einerseits, so zu sagen, am billigsten $\mathrm{zu}$ hahen ist, und andererseits sich innerhalb gewisser Concentrationsgrenzen gegenüher allen Gewebearten indifferent verhält, was z. B. für die Kalisalze nicht gilt. Diese Auffassung lag speciell dem Verfasser als Pflanzenphysiolog um so näher, da es durch Kulturversuche in Nährlösungen sicher festgestellt worden ist, dass Natrium wenigstens für die grosse Mehrzahl der Pflanzen während aller Entwicklungsphasen völlig entbehrlich ist.

Dass diese Ansicht nicht länger haltbar ist, braucht nicht weiter auseinander gesetzt zu werden; es darf vielmehr als unerschütterlich feststehend betrachtet werden, dass neben seinen osmotischen Leistungen dem Chlornatrium oder besser gesagt den Natriumionen 
irgend eine ganz specifische Function bei der Muskelcontraction und bei der Erregungsleitung dureh die Muskelsubstanz (ob auch bei der Erregungsleitung der Nerven?) zukommt. Das Spiel der Geisseln (z. B. der Spermatozoiden) und der Wimperhaare der Flimmerzellen sowohl der Wirbelthiere als auch der Wirbellcsen ist dagedren von der Anwesenheit von Natriumionen in der sie umgebenden Lösung völlig unabhängig und ebenso die Contraction der Stiele der Vorticellen und verwandter Protozoen, obgleich diese Vorgänge sonst mit Vorliebe der Muskelcontraction angereiht werden. - Es wäre von grossem Interesse, das Verhalten der elektrischen Organe, deren Zwischenflüssigkeit durch eine isosmotische Rohrzuckerlösung ersetzt worden ist, zu untersuchen.

Wenn wir nun weiter fragen, in welcher Weise die Natriumsalze bei der Erregungsleitung und der Contraction der Muskeln betheiligt sein könnten, so scheinen sich nur zwei Möglichkeiten darzubieten. Entweder müssen die Natriumsalze einen bestimmten Einfluss auf die Oberflächen-Eigenschaften der Muskelfasern, d. h. auf die oberflächlichste Schicht des Sarkoplasmas (das Sarkolemma kann hier wohl nicht in Betracht kommen) ausüben. dessen Wexfall die Contraction unmöglich macht, ohne dass die Natriumsalze resp. Natriumionen in die Muskelfasern eindringen, oder aber es muss bei der Contraction resp. bei der Erregungsleitung ein yewisser Austausch zwischen Kationen, die sich im Innern der Muskelfasern befinden (also am wabrseheinlichsten von Kaliumionen) und den Natriumionen in der die Muskelfasern umspülenden Lösung stattfinden.

Was die erste Alternative anbetrifft, so ist es sehr schwer sich vorzustellen, wie alle nicht-schädigenden Natriumsalze in äquivalenten Concentrationen genau denselben Finfluss auf die Oberfläche der Muskelfasern (etwa auf die Oberflächenspannung der Sarkoplasmagrenzschicht) ausüben sollten, da dies eine völlige Unwirksamkeit der Anionen und der neutralen Molekeln der Salze auf die betreffenden Eigenschaften der Oberflächeuschicht involvirt. Wäre sie richtig, so würde namentlich zu erwarten sein, dass die minimale Concentration der Natriumionen, die gerade zur Erhaltung der Erregbarkeit ausreicht, eine verschiedene sein würde, je nachdem die Reaction der Zwischenflüssigkeit der Muskeln eine neutrale oder schwach alkalische ist; ein solcher Unterschied wurde aber nicht beobachtet.

Ein gewisser Unterschied sowohl zwischen den optischen als 
auch zwischen den mechanischen Eigenschaften normaler und in Zuckerlösungen unerregbar gewordener Muskeln ist allerdings zu constatiren, ein Unterschied, der namentlich dann recht deutlich zu Tage tritt, wenn man einen Muskel nur bis zur Hälfte seiner Länge in einer Zuckerlösung suspendirt. Die Muskelbälfte, deren Zwischenflüssigkeit entsalzt worden ist, ist entschieden etwas weniger durchscheinend und weniger plastisch als die andere normale Hälfte; doch muss bemerkt werden, dass der Unterschied namentlich in den opiischen Eigenschaften viel geringer ist als zwischen Muskeln, die einerseits in $0,6-0,70_{i 0}$, andererseits in $0,4^{0 /} 0$ Chlornatriumlösungen verweilt haben, obgleich die Muskeln, die in der letzteren Lösung geblieben sind, sich ebensogut contrahiren wie unter normalen Umständen. Vorläufig scheint es mir wahrscheinlich, dass diese Modificationen in dem Aussehen und in der Plasticität von Muskeln, deren $\mathrm{Z}$ wischenflüssigkeit entsalzt worden ist, nur von nebensächlicher Bedeutung sind. Ein eingehenderes Studium der mechanischen Eigenschaften dieser Muskeln, das sobald als möglich in Angriff genommen werden soll, ist allerdings für eine weitere Aufklärung des Gegenstandes unerlässlich. - Im Uebrigen scheint mir namentlich die Thatsache, dass nicht allein das Contractionsvermögen, sondern auch die Fähigkeit der Erregungsleitung aufgehoben wird, sehr gegen die Annahme zu sprechen, dass es sich hier bloss um eine Aenderung der mechanischen Eigenschaften der Muskeln handelt.

Im Ganzen neige ich zur Zeit mehr zu Gunsten der zweiten Alternative, obgleich die mit derselben verbundenen Schwierigkeiten keineswegs zu unterschätzen sind. Wenn sie im Allgemeinen zutreffen sollte, so dürfte es sich wohl lediglich um einen Austausch der Kationen handeln, nicht etwa um einen Uebergang von Natriumsalzen als solchen in die Muskelfasern, denn sonst wäre die Gleichwerthigkeit der verschiedenen Natriumsalze für den Contractionsact der Muskeln kaum verständlich.

Dass zwar die Muskelfasern für die Salze der Alkalien und Erdalkalien, resp. für deren Ionen, nicht in demselben Sinne durchlässig sind, wie etwa für Alkohol, für Glycerin oder für Harnstoff, unterliegt keinem Zweifel; damit ist aber nicht gesagt, dass die Muskelfasern zu allen Zeiten und unter allen Umständen für diese Salze gänzlich undurchäangig sind, - ja es ist ohne Weiteres klar, dass sie während des Wachsthums diese Salze aus der Gewebelymphe anfnehmen müssen. 
Man könnte sich ganz wohl vorstellen, dass in einer bestimmten Phase der Latenzzeit oder des eigentlichen Contractionsvorganges eine solche Aenderung der Oberfläche des Sarkoplasmas stattfindet, dass die Fasern während eines gewissen (wahrscheinlich äusserst kurzen) Zeitraumes für Natrium- und Kaliumionen durchlässig werden. Mir sind thatsächlich bei gewissen Pflanzenzellen (z. B. den Wurzelhaaren ron Trianea bogatensis) Fälle bekannt, wo eine solche nur Bruchtheile einer Secunde danernde Durchlässigkeit des Protoplasts für Salze etc. vorkommt. Ich halte es für nicht unwahrscheinlich, dass die lähmende Wirkung der Kaliumsalze auf die Muskeln mit einer Störung eines solchen Austausches zwischen den Kaliumionen der Muskelfasern und den Natriumionen der Zwischenflüssigkeit zusammenhängt, kann iudessen auf diese Möglichkeit erst in der folgenden Mittheilung eingehen, da sie eine Discussion einer grösseren Anzahl Versuche über die Wirkung von Kalium- und anderen Salzen auf die Muskeln erfordert, die hier zu weit führen würde.

Sehr beachtenswerth ist ferner der Umstand, dass nur Lithiu m salze die Natriumsalze ersetzen können. Natrium und Lithium zeichnen sich unter den Alkali- und Erdalkalimetallen dureh die relative Langsamkeit ihrer Ionenwanderung aus. Durch einen Austausch der einen oder der anderen dieser Ionengattungen mit den Kaliumionen der Muskelfasern könnte also leicht eine elektrische Spannung auftreten, die möglicher Weise eine der Quellen der Actionsströme darstellt.

Auf alle diese Fragen werde ich in späteren Mittheilungen zurückzukommen haben und will daher auf dieselben hier nicht näher eingehen.

Die grösste Schwierigkeit, die der Annahme eines I o ne na usta usches während einer bestimmten Phase der Muskelcontraction oder der Latenzzeit entgegensteht, besteht darin, dass man sich zur Zeit keine rechte Vorstellung darüber bilden kann, wie die in die Muskelfasern übergetretenen Natriumionen aus den Muskelfasern wieder herausgesehafft werden sollen. Bedenkt man z. B., dass die Herzmuskelzellen innerhalb 70 Jahren ca. $24 \times 10^{8}$, und die Athmungsmuskeln ca. $6 \times 10^{5}$ Contractionen ausführen, so leuchtet es ohne Weiteres ein, dass, wenn bei jeder Contraction Natriumionen in die Muskelfasern eindringen und Kalinmionen aus denselben austreten, ein rollständiger Ausgleich zwischen den Concentrationen der Kalium- und Natriumionen in den Muskelfasern und 
den Säften allmählich erfolgen musste, wenn nicht irgend eỉn Mechanismus (im weitesten Sinne des Wortes) einem solchen Ausgleich entgegen wirkte. Thatsächlich sind die Muskelfasern im Alter, so weit bekannt, ebenso reich an Kalium und ebenso arm an Natrium wie in der Jugend.

Man könnte vielleicht geneigt sein, diese letztere Schwierigkeit so hoch zu schätzen, dass sie allein genüge, um die ganze Hypothese eines Ionenaustausches wäbrend der Contraction oder der Fortpflanzung der Erregung unaunehmbar zu machen. Mir scheinen indessen die Schwierigkeiten, welche der Erklärung vieler thatsächlich stattfindenden Stoffwanderungen durch die Drüsenzellen etc. entgegenstehen, vielfach von derselben Ordnung. Unsere Kenntnisse selbst über die seheinbar einfachsten Lebensvorgänge sind eben noch in ihren ersten Anfängen, und wir werden uns nur ganz allmählich bewusst, wie complicirt diese Vorgänge in Wirklichkeit sind. Damit ist aber nicht der geringste Grund für die Annahme gegeben, dass die Gesetze, welche über das physische Geschehen in den Lebewesen obwalten, auch nur um ein Jota von den Gesetzen der anorganischen Natur abweichen. Die Schwierigkeiten, die bei der Erklärung der Lebensvorgänge auftreten, sind vorwiegend bedingt einerseits durch unsere sehr mangelhaften chemischen Kenntnisse über die Bestandtheile des Protoplasmas, andererseits durch das complicirte Ineinandergreifen einer grösseren Anzahl Vorgänge in einem System von sehr geringer räumlicher Ausdehnung, was der Analyse des Gesanmtgeschehens in seine einzelnen Componenten und der zeitlichen Verfolgung dieser letzteren grosse Hindernisse entgegensetzt. Die bisberige Geschichte der Naturwissenschaften im Allgemeinen und der Physiologie im Besonderen lässt hoffen, dass auch diese Schwierigkeiten durch glückliche Fragestellungen und den Fortschritt der physiologischen Methodik allmählich überwunden werden.

\section{Zusammenfassung der wichtigsteu Ergebnisse.}

1. Werden Muskeln in mit dem Blute isosmotische Lösungen vou Rohrzucker oder von anderen Non-Elektrolyten, für welche die Muskelfasern undurchlässig oder nur langsam durchlässig sind, gesetzt, so verlieren sie nach einiger Zeit das Vermögen, sich zu contrahiren und Erregungen fortzupflanzen. 
2. Diese Unerregbarkeit der Muskeln ist durch die Exosmose des Chlornatriums aus der Zwischenflüssigkeit der Muskeln, d. h. aus der Lösung, welche die einzelnen Muskelfasern umspülen, verursacht.

3. Die Nervenstämme verlieren ihre Erregbarkeit durch Verweilen in reinen Zuckerlösungen etc. nicht; doch ist es nicht ausgeschlossen, dass unter diesen Umständen zwischen dem Axencylinder und dem Nervenmark eine Chlornatriumlösung zurückbleibt, soferne ein ly $\mathrm{mph}$ eführender periaxialer Ra um um den Axencylinder wirklich existirt.

4. Die Erregbarkeit von Muskeln, die durch längeres Verweilen in Zuckerlösungen etc. unreizbar geworden sind, kehrt allmählich wieder zurück nach Zusatz einer geringen Menge von Chlornatrium zu den betreffenden Lösungen.

5. Der unerregbare Zustand ist $n \mathrm{ich}$ t dadurch veranlasst, dass in Folge des Chlornatriumentzuges der elektrische Widerstand der Muskeln erhöht wird, da die Muskeln ebensogut gegen mechanische wie gegen elektrische Reize unempfindlich sind, und da bei der allmählichen Verarmung der Zwischenflüssigkeit der Muskeln an Chlornatrium die Erregbarkeit der Muskeln zunächst (bis die Concentration dieses Salzes auf ca. 0,12\% gesunken ist) nur sehr wenig abnimmt, bei etwas weiterer Verarmung an Chlornatrium dagegen sehr rasch eine enorme Abnahme erfährt und bald völlig erlischt.

6. In $6 \%$ Rohrzuckerlösungen etc, dieca. $0,1-0,12 \%$ $\mathrm{NaCl}$ enthalten, bleiben Muskeln ungefähr ebenso lange erregbar wie in $0,6-0,7 \%$ Chlornatrium.

7. Der niedrigste Chlornatriumgehalt einer Lösung, der eben ausreicht, um die Muskeln merklich erregbar zu halten, beträgt $0,07 \pm 0,003 \%$ (bei Temperaturen von $16-22^{\circ} \mathrm{C}$.).

8. Das Chlornatrium kann durch alle nicht-giftigen Natriumsalze ersetzt werden, wobei die minimalen Concentrationen aller dieser Salze, die zur Erhaltung der Erregbarkeit eben ausreichen, ungefähr mit einer $0.07 \%$ igen $\mathrm{NaCl}$ äquivalent sind. Es kommt also bei der Erhaltung der Erregbarkeit von Muskeln fast sicher lediglich auf die Natriumionen an, während die Anionen (und elektrisch neutralen Molekeln) sich passiv verhalten.

9. Natriumsalze können durch Lithinmsalze ersetzt werden, nicht dagegen durch Kalium-, Rubidium-, 
386 E. Overton: Beiträge zur allgem. Nuskel- und Nervenphysiologie.

Caesium-oder Ammoniumsalze, und ebensowenig durch Magnesium-, Calcium-, Strontium oder Baryumsalze. Die Minimalconcentrationen der Lithinmsalze, die eine Contraction ermöglichen, sind die gleichen wie bei den entsprechenden Natriumsalzen, wenn man nach Molekülconcentrationen rechnet, geringer dagegen, wenn man nach Gewichtsprocenten zählt.

10. Die Rolle, welche die Natrium- resp. die Lithiumionen bei der Fortpflanzung der Erregung und bei der Contraction der Muskeln spielt, ist noch nicht aufgeklärt; vielleicht findet während dieser Vorgänge ein gewisser Austausch zwischen den Kaliumionen der Muskelfasern und den Natriumi onen der sie umspülenden Lösung statt, doch ist eine solche Annahme mit beträchtlichen Schwierigkeiten verbunden. 